\title{
Crystallographic analysis and phase field simulation of transformation plasticity in a multifunctional $\beta$-Ti alloy
}

\author{
Jiaming Zhu ${ }^{\text {a,b }}$, Honghui Wu ${ }^{\mathrm{b}}$, Dong Wang ${ }^{\mathrm{a}}$, Yipeng Gao ${ }^{\mathrm{c}}$, Haoliang Wang ${ }^{\mathrm{d}}$, Yulin Hao ${ }^{\mathrm{d}}$, Rui \\ Yang $^{\mathrm{d}}$, Tong-Yi Zhang ${ }^{\mathrm{e}, *}$, Yunzhi Wang ${ }^{\mathrm{a}, \mathrm{c}, *}$ \\ ${ }^{a}$ Center of Microstructure Science, Frontier Institute of Science and Technology, Xi'an Jiaotong University, \\ Xi'an 710049, China \\ ${ }^{\mathrm{b}}$ Department of Mechanical Engineering, Hong Kong University of Science and Technology, Clear Water Bay, \\ Kowloon, Hong Kong, China \\ ${ }^{\mathrm{c}}$ Department of Materials Science and Engineering, The Ohio State University, 2041 College Road, Columbus, \\ OH 43210, USA \\ ${ }^{d}$ Shenyang National Laboratory for Materials Science, Institute of Metal Research, Chinese Academy of \\ Sciences, 72 Wenhua Road, Shenyang 110016, China \\ e Shanghai University Materials Genome Institute and Shanghai Materials Genome Institute, Shanghai \\ University, 99 Shangda Road, Shanghai 200444, China
}

\begin{abstract}
The gum-like multifunctional $\beta$-Ti alloy Ti-24Nb-4Zr-8Sn-0.10O (in wt.\%), known as Ti2448, has attracted a lot of attention lately for promising biomedical applications due to its ultralow apparent elastic modulus, high strength, super-elasticity, strong fatigue resistance, and excellent biocompatibility. In this study we investigate the deformation mechanisms in association with martensitic transformation (MT) in the alloy. Using a combination of crystallographic analysis and phase field simulation we find a rich variety of strain-accommodating domain patterns, including 30 twinning modes and 6 herringbone structures. These diverse strain accommodating modes, especially the excellent geometrical compatibilities of the twins and herringbone structures, provide the MT with great flexibility to adapt to arbitrary shape changes and defect structures generated during plastic deformation, which may have contributed to the exceptional mechanical properties of the alloy.
\end{abstract}

Keywords: A. Strain accommodation, Geometrical compatibility; A. Transformation twins; A. Herringbone structure; C. Computer simulation; B. GUM metals 


\section{Introduction}

The so-called gum metals have been shown to have many unprecedented properties including ultralow apparent elastic modulus, super-elasticity, high strength, strong fatigue resistance, outstanding elongation to failure, Invar and Elinvar anomalies, and excellent biocompatibility (Y.L. Hao et al., 2007; Morris Jr. et al., 2010; Saito et al., 2003). In particular, the multifunctional $\beta$-Ti alloy, Ti2448 (Ti-24Nb-4Zr-8Sn-0.10O in wt.\%) (Guo et al., 2009; Y.L. Hao et al., 2007; Hao et al., 2005; Long and Rack, 1998; S. Q. Zhang et al., 2011), has an apparent elastic modulus as low as $40 \mathrm{GPa}$, which is much lower than that of the currently used Ti-6Al4V orthopedic implants (elastic modulus $110 \mathrm{GPa}$ (Long and Rack, 1998) and closer to that of human bones ( 20 GPa) (Zysset et al., 1999) and thus much more effective in alleviating the "stress shielding" effect for orthopedic application (Geetha et al., 2009; Long and Rack, 1998; Saito et al., 2003). However, the deformation mechanisms underlying these unique properties are still far from clear (Hao et al., 2012). For example, Saito et al (Saito et al., 2003) proposed a dislocation-free deformation mechanism. Latter Cui et al. (Cui et al., 2009) proposed a set of reversible deformation mechanisms including dislocation loop nucleation and growth and $\mathrm{BCC}$ to $\alpha$ " martensitic transformation (MT). Based on their transmission electron microscopy observations, Plancher et al. (Plancher et al., 2013) demonstrated that a phase-transformationassisted mechanical twinning mechanism governs the plasticity in the GUM metal without direct involvement of dislocations. Most recently Liu et al. (Liu et al., 2013) suggested that reversible dual martensitic transformations (MTs) of B2 to $\alpha$ " and BCC to $\delta$ induced by a $\mathrm{Nb}$ concentration modulation in the BCC phase are responsible for the nonlinear elastic behavior of Ti2448. Wang and Sehitoglu (Wang and Sehitoglu, 2014) reported that an internally twinned martensitic structure shows an apparent modulus two times smaller than that of a single variant structure. Moreover, the oxygen content, which can change the transformation strain, is also found to have a significant impact on the elastic behavior of the alloy through its effect on the MT (Obbard et al., 2011). Thus more and more observations seem to indicate strongly that the various exceptional mechanical properties found in Ti2448 are closely related to, although may not be limited to, the MT. But a detailed description of such MT in the system is not available. Therefore, a systematic analysis of MT in Ti2448 becomes indispensable to understand both elastic and plastic deformation of the alloy. 
Plastic deformation of crystals can be realized by the mobilization and multiplication of dislocations, structural phase transformations or both. Although dislocations are the dominant carriers of plastic strain in most crystalline materials, structural phase transformations are also nature carriers of plastic strain in many material systems including shape memory alloys (SMAs), steels and, most recently, GUM metals, to name a few. In the latter it is actually the interplay and coupling between structural transformations and dislocations that determine their mechanical behavior. Consider for example martensitic transformations (MTs) where the symmetry breaking during the transformation creates multiple deformation pathways and strain domains. If the domain structures are "flexible enough" to be fully compatible with the parent phase, then the MTs could be induced easily either by temperature or stress and be able to accommodate various kinds of deformations without involving much dislocation activities when the applied strain does not exceed the maximum transformation strain (Chen et al., 2013; James et al., 2005). Unfortunately strains generated by MTs in most structural materials and SMAs including NiTi cannot be fully self-accommodated and abundant dislocations are generated during the transformations (Bowers et al., 2014; Delville et al., 2011; Eggeler et al., 2004; Norfleet et al., 2009; Paranjape et al., 2016; Pelton et al., 2012; Song et al., 2013; Zarnetta et al., 2010). However, the MT in Ti2448 seems to be quite different from those found in conventional SMAs and $\beta$-Ti alloys and the dislocation content is rather low in plastically deformed samples (Y. L. Hao et al., 2007).

It will be shown in this study that the transformation strain in Ti2448 can be self-accommodated by a plethora of martensitic domain structures including single variants without any lattice invariant shear, 6 types of compound twins, 12 type-I twins and 12 type-II twins, which yield total of 108 invariant habit planes of martensitic plates, 2 types of herringbone structures and 2 types of wedge-structures. Such an excellent geometrical compatibility may provide the system with great flexibility to accommodate arbitrary deformation and structural defects, leading to its exceptional mechanical properties. Below (Section 2) we will first present a systematic crystallographic analysis of self-accommodating domain patterns of martensite in Ti2448 using the phenomenological theory of martensite crystallography (PTMC) (Bhattacharya, 2003; Bowles and Mackenzie, 1954; Mackenzie and Bowles, 1954; Wechsler et al., 1953), followed by 
(Sectiton 3) phase field simulations of microstructure evolution during the cubic to orthorhombic MT in the alloy based on a combination of Landau theory (Landau and Lifshitz, 1980) and Khachaturyan-Shatalov's microelasticity theory (KS theory) (Khachaturyan, 1983) of structural transformations. To validate the PTMC and phase field simulation predictions, twin structures produced by stress-induced martensitic transformation in Ti2448 are characterized experimentally using high-resolution scanning transmission electron microscopy, as detailed in Section 4. The results are analyzed in Section 5 and, in particular, the geometrical compatibility of the GUM metal is compared with that of the $\mathrm{Cu}_{68} \mathrm{Zn}_{15} \mathrm{Al}_{17}$ (James et al., 2005) in terms of their fulfillment of the so-called co-factor conditions. The excellent geometrical compatibility and relatively small transformation strain of martensite in Ti2448 make the local stresses associated with the martensitic domain structures about one order of magnitude smaller than those found in the NiTi-based commercial SMAs. Finally the major findings are summarized in Section 6.

\section{Crystallographic analysis}

\subsection{Formulation of the transformation matrix}

MT is a diffusionless structural phase transition that allows one to delineate it with a transformation matrix (also referred to as deformation gradient matrix) that maps the lattice of the parent phase onto that of the product phase. Below we first formulate the transformation matrix for the MT in Ti2448.

According to the lattice correspondence (LC) for the $\beta$ (BCC, point group $m \overline{3} m$ ) to $\alpha$ " (orthorhombic, point group $\mathrm{mmm}$ ) MT in Ti2448

$$
[001]_{\beta} \rightarrow[100]_{\alpha}{ }^{\prime \prime},[1 \overline{1} 0]_{\beta} \rightarrow[010]_{\alpha}{ }^{\prime \prime},[110]_{\beta} \rightarrow[001]_{\alpha}{ }^{\prime \prime},
$$

and the symmetry operations in point groups that preserve such a LC, only 8 operations (out of the 48 symmetry operations in $m \overline{3} m$ ) (Hahn, 2006) are left after the transformation, leading to 6 $(=48 / 8)$ crystallographic equivalent deformation modes (or correspondence variants or deformation variants) (Gao et al., 2016) characterized by 6 different transformation strain tensors. 
In fact, because internal shuffle is also involved during the transformation, which doubles the deformation variants, there are total 12 deformation variants (Gao et al., 2014). The two deformation variants with different internal shuffles but producing the same transformation strain will not be distinguished in the current study. We choose the three orthogonal axes of the cubic crystal of the parent phase as the reference coordinate system, as shown in Fig. 1(a). Following the Bain correspondence and using the lattice parameters of the austenite and martensite (Liu et al., 2013) given in both Fig. 1 and Table 1, the transformation matrices that map the parent phase lattice onto that of the corresponding martensitic variants read

$$
\begin{aligned}
\boldsymbol{U}_{1} & =\left[\begin{array}{ccc}
0.9770 & 0 & 0 \\
0 & 1.0136 & -0.0108 \\
0 & -0.0108 & 1.0136
\end{array}\right], \boldsymbol{U}_{2}=\left[\begin{array}{ccc}
0.9770 & 0 & 0 \\
0 & 1.0136 & 0.0108 \\
0 & 0.0108 & 1.0136
\end{array}\right], \\
\boldsymbol{U}_{3} & =\left[\begin{array}{ccc}
1.0136 & 0 & -0.0108 \\
0 & 0.9770 & 0 \\
-0.0108 & 0 & 1.0136
\end{array}\right], \boldsymbol{U}_{4}=\left[\begin{array}{ccc}
1.0136 & 0 & 0.0108 \\
0 & 0.9770 & 0 \\
0.0108 & 0 & 1.0136
\end{array}\right], \\
\boldsymbol{U}_{5} & =\left[\begin{array}{ccc}
1.0136 & -0.0108 & 0 \\
-0.0108 & 1.0136 & 0 \\
0 & 0 & 0.9770
\end{array}\right], \boldsymbol{U}_{6}=\left[\begin{array}{ccc}
1.0136 & 0.0108 & 0 \\
0.0108 & 1.0136 & 0 \\
0 & 0 & 0.9770
\end{array}\right] .
\end{aligned}
$$

Table 1 Lattice parameters and elastic constants of austenite and martensite at room temperature (Liu et al., 2013; Y. W. Zhang et al., 2011).

\begin{tabular}{ccccccc}
\hline $\mathrm{a}_{\beta}(\AA)$ & $\mathrm{a}_{\alpha^{\prime \prime}}(\AA)$ & $\mathrm{b}_{\alpha^{\prime \prime}}(\AA)$ & $\mathrm{c}_{\alpha^{\prime \prime}}(\AA)$ & $\mathrm{C}_{11}(\mathrm{GPa})$ & $\mathrm{C}_{12}(\mathrm{GPa})$ & $\mathrm{C}_{44}(\mathrm{GPa})$ \\
\hline 3.298 & 3.222 & 4.788 & 4.667 & 57.2 & 36.1 & 35.9 \\
\hline
\end{tabular}

\subsection{Invariant habit plane formed by a single martensitic variant}

Kinematic compatibility is required for an invariant habit plane between the martensite and austenite. In general, lattice invariant deformations are needed to form an invariant habit plane; however, when the transformation strain of a single variant is an invariant plane strain, lattice invariant deformation becomes unnecessary (Wayman, 1994). The eigenvalues of the transformation matrices in Eq. (1) are 0.977, 1.003, and 1.024, which means that the stress-free transformation strain (SFTS) is an invariant plane strain by itself and a single deformation 
variant of the martensite has an invariant habit plane satisfying the following equation (Bhattacharya, 2003; Bowles and Mackenzie, 1954; Wechsler et al., 1953)

$$
Q U_{j}-I=b \otimes m
$$

where $\boldsymbol{Q}$ is a rigid body rotation of variant $j$ with respect to the parent phase, $\boldsymbol{U}_{j}$ is the transformation matrix of variant $j$ given in Eq. (1), $\boldsymbol{Q} \boldsymbol{U}_{j}$ is the invariant plane strain, $\boldsymbol{I}$ is the identity matrix, $\boldsymbol{m}$ is the normal of the invariant plane, $\boldsymbol{b}$ is the displacement that characterizes the shape change (uniform lattice distortion) associated with the invariant plane strain, and $\otimes$ represents dyadic product of two vectors. The solutions of Eq. (2) for the six deformation variants are listed in Table 2. It should be pointed out that our predictions of the signle variant habit planes agree well with those observed by TEM in Ti2448 (Yao et al., 2016).

Table 2 solutions of Eq. (2).

\begin{tabular}{ccc}
\hline Variant NO. & $\boldsymbol{b}$ & $\boldsymbol{m}$ \\
\hline \multirow{2}{*}{1} & $(0.03367,-0.02366,0.02366)$ & $(-0.6925,-0.5101,0.5101)$ \\
& $(0.03367,0.02366,-0.02366)$ & $(-0.6925,0.5101,-0.5101)$ \\
2 & $(0.03367,0.02366,0.02366)$ & $(-0.6925,0.5101,0.5101)$ \\
& $(0.03367,-0.02366,-0.02366)$ & $(-0.6925,-0.5101,-0.5101)$ \\
3 & $(-0.02366,0.03367,0.02366)$ & $(-0.5101,-0.6925,0.5101)$ \\
& $(0.02366,0.03367,-0.02366)$ & $(0.5101,-0.6925,-0.5101)$ \\
4 & $(0.02366,-0.03367,0.02366)$ & $(0.5101,0.6925,0.5101)$ \\
& $(-0.02366,-0.03367,-0.02366)$ & $(-0.5101,0.6925,-0.5101)$ \\
5 & $(-0.02366,0.02366,0.03367)$ & $(-0.5101,0.5101,-0.6925)$ \\
& $(0.02366,-0.02366,0.03367)$ & $(0.5101,-0.5101,-0.6925)$ \\
& $(0.02366,0.02366,0.03367)$ & $(0.5101,0.5101,-0.6925)$ \\
& $(-0.02366,-0.02366,0.03367)$ & $(-0.5101,-0.5101,-0.6925)$ \\
\hline
\end{tabular}


(a)

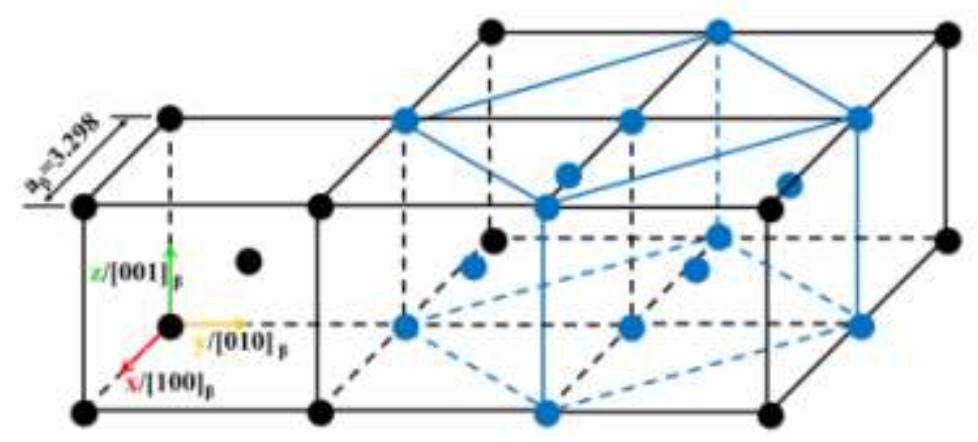

(b)

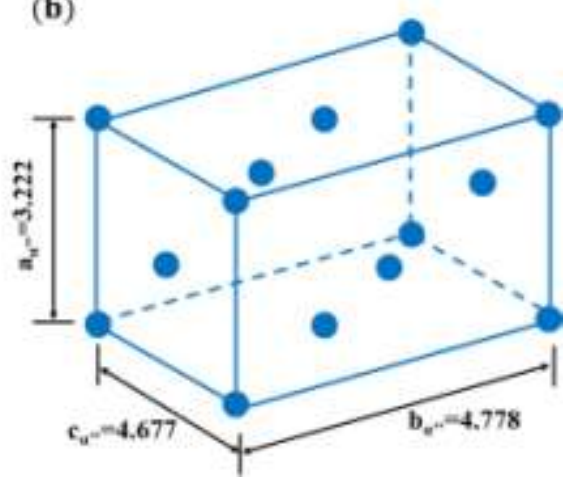

Fig. 1. Crystal lattice of (a) parent (BCC) and (b) martensitic (orthorhombic) phase, respectively. The structure outlined in blue in (a) notes the Bain correspondence between the parent and martensitic phases. Lattice parameters in this figure have a unit of angstrom. 


\subsection{Twinning modes}

Similar to the invariant plane between martensite and austenite, a twin plane is an invariant plane between two martensitic variants. A twin plane between variant $i$ and variant $j$ needs to comply with the following kinematic compatibility condition (Bhattacharya, 2003; Bowles and Mackenzie, 1954; Wechsler et al., 1953)

$$
\boldsymbol{Q} \boldsymbol{U}_{j}-\boldsymbol{U}_{i}=\boldsymbol{a} \otimes \boldsymbol{n}
$$

where $\boldsymbol{Q}$ is a rigid body rotation of variant $j$ with respect to variant $i, \boldsymbol{a}$ is a shear vector and $\boldsymbol{n}$ is the twin plane normal. With the transformation matrices given in Eq. (1), 30 solutions can be obtained from Eq. (3), which, after Gurtin (Gurtin, 1983), can be categorized into three types of twins, i.e., 6 compound twins, 12 type-I twins, and 12 type-II twins. Compound twin can be formed by variants that have a mirror symmetry, such as $\boldsymbol{n}=(0,1,0)$ between variants 1 and $2, \boldsymbol{n}$ $=(0,0,1)$ between variants 3 and 4 , and $\boldsymbol{n}=(1,0,0)$ between variants 5 and 6 . Type-I and typeII twins can be found between variants that have no mirror symmetry, for instance, variants 1 and 3, 1 and 4, 1 and 5, and 1 and 6. Type-I twin planes are rational, for example $\boldsymbol{n}=(-1,1,0)$ between variants 1 and 3. In contrast, type-II twin planes are irrational, for example $\boldsymbol{n}=(0.6508$, $0.6508,-0.3911)$ between variants 1 and 3. All the twin planes are listed in appendix B.

\subsection{Invariant habit planes of internally twinned martensitic particles}

Twinning can not only render martensitic variants compatible with each other but also produce invariant habit planes between an internally twinned martensitic plate and the austenite. An invariant habit plane formed by the internally twinned martensitic variants should satisfy the following equations (Bhattacharya, 2003; Bowles and Mackenzie, 1954; Wechsler et al., 1953)

$$
\begin{gathered}
\boldsymbol{Q} \boldsymbol{U}_{j}-\boldsymbol{U}_{i}=\boldsymbol{a} \otimes \boldsymbol{n} \\
\boldsymbol{Q}^{\prime}\left[\lambda \boldsymbol{Q} \boldsymbol{U}_{j}+(1-\lambda) \boldsymbol{U}_{i}\right]=\boldsymbol{I}+\boldsymbol{b} \otimes \boldsymbol{m}
\end{gathered}
$$

where $\boldsymbol{Q}^{\prime}$ is a rigid body rotation of the martensitic variants with respect to the parent phase and 
$\lambda$ is the volume fraction of variant $j$.

From Section 2.2 we have learned that there are three types of twins, of which the compound twins cannot satisfy Eq. (4b) and, thus, cannot form invariant habit planes. Both type-I and typeII twins have 48 options to form invariant habit planes. Taking the combination of variants 2 and 3 as an example, invariant habit planes that they can form are listed in Table 3.

Table 3 Habit plane of internally twinned martensitic plates consisting of variants 2 and 3

\begin{tabular}{cccc}
\hline Twinning type & Twin plane & $\begin{array}{c}\lambda \text { (volume fraction of } \\
\text { variant } 3)\end{array}$ & Habit plane \\
\hline Type-I & $(-1,-1,0)$ & 0.2293 & $(-0.7099,0.4051,0.5761)$ \\
Type-I & $(-1,-1,0)$ & 0.2293 & $(-0.6083,-0.3700,-0.7022)$ \\
Type-I & $(-1,-1,0)$ & 0.7707 & $(-0.3700,-0.6083,0.7022)$ \\
Type-I & $(-1,-1,0)$ & 0.7707 & $(0.4051,-0.7099,-0.5761)$ \\
Type-II & $(0.6508,-0.6508,-0.3911)$ & 0.2413 & $(-0.7030,0.4162,0.5766)$ \\
Type-II & $(0.6508,-0.6508,-0.3911)$ & 0.2413 & $(-0.6104,-0.3402,-0.7153)$ \\
Type-II & $(0.6508,-0.6508,-0.3911)$ & 0.7587 & $(-0.3402,-0.6104,0.7153)$ \\
Type-II & $(0.6508,-0.6508,-0.3911)$ & 0.7587 & $(0.4162,-0.7030,-0.5766)$ \\
\hline
\end{tabular}

\subsection{Crossing twins}

The herringbone structure is a common example of crossing twins in which four different variants meet at a quadruple junction. The kinematic compatibility requirement can be written as (Bhattacharya, 2003)

$$
\begin{aligned}
& \boldsymbol{Q}_{1} \boldsymbol{U}_{j}-\boldsymbol{U}_{i}=\boldsymbol{a}_{1} \otimes \boldsymbol{n}_{1} \\
& \boldsymbol{Q}_{2} \boldsymbol{U}_{k}-\boldsymbol{U}_{j}=\boldsymbol{a}_{2} \otimes \boldsymbol{n}_{2} \\
& \boldsymbol{Q}_{3} \boldsymbol{U}_{l}-\boldsymbol{U}_{k}=\boldsymbol{a}_{3} \otimes \boldsymbol{n}_{3} \\
& \boldsymbol{Q}_{4} \boldsymbol{U}_{i}-\boldsymbol{U}_{l}=\boldsymbol{a}_{4} \otimes \boldsymbol{n}_{4} \\
& \boldsymbol{Q}_{1} \boldsymbol{Q}_{2} \boldsymbol{Q}_{3} \boldsymbol{Q}_{4}=\boldsymbol{I}
\end{aligned}
$$




$$
\boldsymbol{n}_{1}, \boldsymbol{n}_{2}, \boldsymbol{n}_{3} \text { and } \boldsymbol{n}_{4} \text { lie on a plane. }
$$

For Ti2448, 6 solutions corresponding to two kinds of herringbone structures can be obtained from Eq. (5) as shown in Fig. 2. Figures 2(a)-(c) are one kind of herringbone structure where the midribs (black lines in the middle of the herringbone structures) are compound twins, and twins on the two sides are type-II twins. This kind of structure will be called C-II herringbone structure hereafter. Figures 2(d)-(f) belong to another kind of herringbone structure whose midribs are type-I twins and the two sides are compound twins. This kind of structure will be termed as I-C herringbone structure hereafter. These herringbone structures, as important deformation carriers, have been observed in TEM characterization of Ti2448 (Y.L. Hao et al., 2007). 

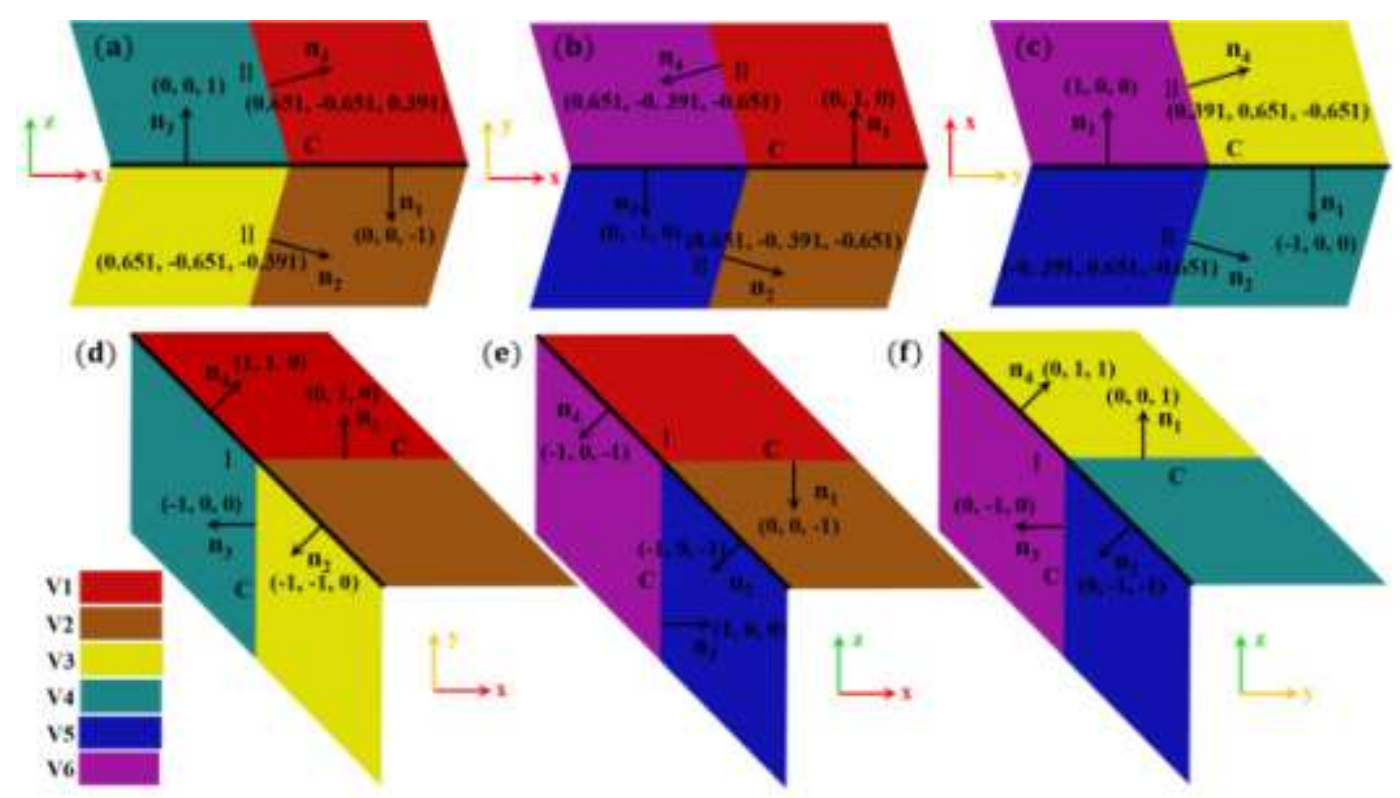

Fig. 2. Schematic drawing of herringbone structures obtained from solutions of Eq. (5). $\mathrm{Vj}(j=1-6)$ is short for variant $j, \mathrm{C}$ denotes compound twin, and I and II represent type-I and type-II twins, respectively. 


\subsection{Wedge-like microstructure}

As shown schematically in Fig. 3, the wedge-like polytwin microstructure is another type selfaccommodating polydomain structure, which satisfies three compatibility conditions: (i) the red and yellow, and purple and blue are respectively twin related variant pairs; (ii) the twins formed by these two pairs of variants should be compatible with the parent phase and (iii) with each other. These three compatibility conditions can be written as

$$
\begin{aligned}
& \boldsymbol{Q}_{1} \boldsymbol{U}_{j}-\boldsymbol{U}_{i}=\boldsymbol{a}_{1} \otimes \boldsymbol{n}_{1} \\
& \boldsymbol{Q}_{2} \boldsymbol{U}_{l}-\boldsymbol{U}_{k}=\boldsymbol{a}_{2} \otimes \boldsymbol{n}_{2} \\
& \boldsymbol{Q}_{3}\left(\boldsymbol{U}_{i}+\lambda_{1} \boldsymbol{a}_{1} \otimes \boldsymbol{n}_{1}\right)=\boldsymbol{I}+\boldsymbol{b}_{1} \otimes \boldsymbol{m}_{1} \\
& \boldsymbol{Q}_{4}\left(\boldsymbol{U}_{k}+\lambda_{2} \boldsymbol{a}_{2} \otimes \boldsymbol{n}_{2}\right)=\boldsymbol{I}+\boldsymbol{b}_{2} \otimes \boldsymbol{m}_{2} \\
& \boldsymbol{b}_{1} \| \boldsymbol{b}_{2}, \text { but } \boldsymbol{m}_{1} \sharp \boldsymbol{m}_{2}
\end{aligned}
$$

where $\lambda_{1}$ and $\lambda_{2}$ are volume fractions of variants $j$ and $l$, respectively. Equations (6a) and (6b), (6c) and (6d), and (6e) correspond to condition (i), (ii) and (iii), respectively. In Ti2448, it is found that the compound twins and type-I and type-II twins cannot satisfy exactly Eq. (6). Combinations of variants that are closest to the solution of Eq. (6) are variants 1, 3, 4, 6, and variants $2,3,4,5$, whose $\boldsymbol{b}_{1}$ deviates from $\boldsymbol{b}_{2}$ by $4.1^{\circ}$. Detailed information on these combinations is listed in Table 4. The wedge-like structures are not expected in a defect-free crystal. However, these structures could be expected in defect-bearing systems, where the deviation between $\boldsymbol{b}_{1}$ and $\boldsymbol{b}_{2}$ could be offset by defects.

Table 4 Combinations that most likely to form a wedge-like structure in Ti2448

\begin{tabular}{cccc}
\hline $\begin{array}{c}\text { Variant } \\
\text { combinations }\end{array}$ & Volume ratio & $\boldsymbol{n}$ (twin planes) & $\boldsymbol{m}$ (habit planes) \\
\hline \multirow{2}{*}{$1,3,4,6$} & $\mathrm{~V} 1: \mathrm{V} 3=0.7707: 0.2293$ & $\mathrm{~V} 1 \& \mathrm{~V} 3(-1,1,0)$ & $\mathrm{V} 1 \& \mathrm{~V} 3(-0.6083,0.3700,-0.7022)$ \\
& $\mathrm{V} 4: \mathrm{V} 6=0.2293: 0.7707$ & $\mathrm{~V} 4 \& \mathrm{~V} 6(0,1,-1)$ & $\mathrm{V} 4 \& \mathrm{~V} 6(-0.5761,-0.4051,-0.7099)$ \\
& $\mathrm{V} 2: \mathrm{V} 3=0.7707: 0.2293$ & $\mathrm{~V} 2 \& \mathrm{~V} 3(-1,-1,0)$ & $\mathrm{V} 2 \& \mathrm{~V} 3(-0.6083,-0.3700,-0.7022)$ \\
$2,3,4,5$ & $\mathrm{~V} 4: \mathrm{V} 5=0.2293: 0.7707$ & $\mathrm{~V} 4 \& \mathrm{~V} 5(0,-1,-1)$ & $\mathrm{V} 4 \& \mathrm{~V} 5(-0.5761,0.4051,-0.7099)$ \\
\hline
\end{tabular}




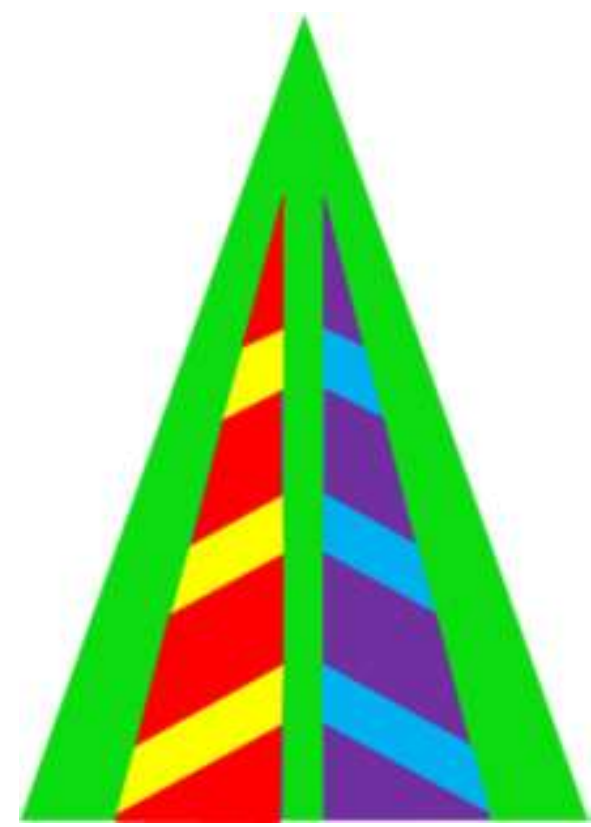

Fig. 3. Schematic drawing of a wedge-like self-accommodating microstructure consisting of 4 martensitic variants represented by the red, yellow, purple and blue colors. The austenite is represented by the green color. 
The above crystallographic analysis based on PTMC predicts ample building blocks for selfaccommodating martensitic domain structures in Ti2448, including single variant, compound twins, type-I and type-II twins, crossing twins, and wedge-like internally twinned structures. Such a rich variety of building blocks provides a strong ability for the MT in the system to selfaccommodate as well as to adapt to existing defects without generating new dislocations.

\section{Phase field simulation}

The above crystallographic analysis based on PTMC is solely a geometrical compatibility analysis. In order to provide direct predictions on the detailed microstructure evolution process and formation of various self-accommodating domain patterns discussed above, with local elastic distortions allowed, we employ the phase field method (Chen, 2002; Wang and Li, 2010), which has been applied successfully to MTs in various materials systems (Artemev et al., 2000; Finel et al., 2010; Gao et al., 2014; Jin et al., 2001; Levitas, 2013; Lloveras et al., 2010; Mamivand et al., 2014; MILITZER et al., 2006; Moelans et al., 2008; Pi et al., 2016; Wang and Khachaturyan, 1997; Yeddu et al., 2013).

\subsection{Stress free transformation strain (SFTS) calculation}

The SFTS (i.e. eigenstrain) of the $p$-th variant of martensite in Ti2448 can be calculated directly from the transformation matrices formulated in Eq. (1) according to the following equation

$$
\varepsilon_{i j}^{S F}(p)=\frac{1}{2}\left(\boldsymbol{U}_{p}^{T} \boldsymbol{U}_{p}-\boldsymbol{I}\right),(p=1 \sim 6),
$$

where the superscript $T$ indicates matrix transpose, and $\boldsymbol{I}$ is the identity matrix.

\subsection{Free energy formulation}

In the phase field approach, the parent and martensitic phases in Ti2448 can be characterized using six non-conserved structural order parameters, $\eta_{p} \quad(p=1 \sim 6)$, with $\left(\eta_{p=1 \sim 6}=0\right)$

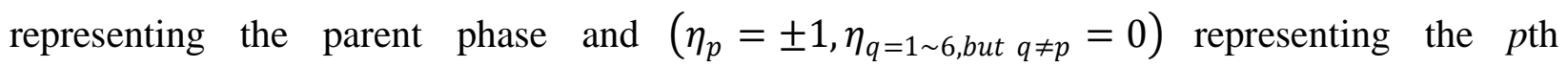
correspondence variant of the martensitic phase, where +1 and -1 denote atomic shuffle in two 
opposite directions in a correspondence variant. The total free energy, $F$, of the system can be formulated as the following

$$
F=\int\left[f_{c h}\left(\eta_{1}, \cdots \eta_{6}\right)+f_{g r}\left(\eta_{1}, \cdots \eta_{6}\right)\right] d^{3} \boldsymbol{r}+E_{e l},
$$

where the local chemical free energy is approximated by a sixth-order Landau polynomial (Wang et al., 2014; Wang and Khachaturyan, 1997)

$$
f_{c h}\left(\eta_{1}, \cdots \eta_{6}\right)=\frac{1}{2} A_{1} \sum_{p=1}^{6} \eta_{p}^{2}(\boldsymbol{r})-\frac{1}{4} A_{2} \sum_{p=1}^{6} \eta_{p}^{4}(\boldsymbol{r})+\frac{1}{6} A_{3}\left(\sum_{p=1}^{6} \eta_{p}^{2}(\boldsymbol{r})\right)^{3},
$$

with $A_{1}=A_{0}\left(T-T_{0}\right), A_{2}$ and $A_{3}$ being chemical free energy coefficients. The non-local gradient energy associated with spatial non-uniformity of the structural order parameters, $\eta_{p}$, can be approximated by (Wang and Khachaturyan, 1997)

$$
f_{g r}\left(\eta_{1}, \cdots \eta_{6}\right)=\frac{1}{2} \beta \sum_{p=1}^{6}\left(\nabla \eta_{p}\right)^{2}
$$

with $\beta$ being the gradient energy coefficient. The stress-free transformation strain field associated with the MT can be formulated as a function of the structural order parameters:

$$
\varepsilon_{i j}^{M T}(\boldsymbol{r})=\sum_{p=1}^{6} \varepsilon_{i j}^{S F}(p) \eta_{p}^{2}(\boldsymbol{r})
$$

where $\boldsymbol{r}$ is the spatial coordinate. The coherency elastic strain energy originating from the lattice misfit between the parent and martensitic phases and among different martensitic variants is formulated based on Khachaturyan-Shatalov's microelasticity theory (KS-theory) (Khachaturyan, 1983)

$$
E_{e l}=\frac{1}{2} \sum_{p=1}^{6} \sum_{q=1}^{6} \int \frac{d^{3} k}{(2 \pi)^{3}} B_{p q}(\boldsymbol{n})\left\{\eta_{p}^{2}\right\}_{k}\left\{\eta_{q}^{2}\right\}_{k}^{*}
$$

where the integral is taken in the reciprocal space, $\boldsymbol{n}=\frac{\boldsymbol{k}}{k}$ is a unit vector and $\boldsymbol{k}$ is a vector with 
modulus $k$ in the reciprocal space, $\left\{\eta_{p}^{2}\right\}_{k}$ represents the Fourier transformation of $\eta_{p}^{2},\left\{\eta_{q}^{2}\right\}_{k}^{*}$ is the complex conjugate of $\left\{\eta_{q}^{2}\right\}_{k}$. For a system whose boundaries are fixed (e.g., a grain in a polycrystal), $B_{p q}(\boldsymbol{n})$ can be written as (Khachaturyan, 1983)

$$
B_{p q}(\boldsymbol{n})=\left\{\begin{array}{ll}
C_{i j k l} \varepsilon_{i j}^{S F}(p) \varepsilon_{k l}^{S F}(q) & \boldsymbol{n}=0 \\
C_{i j k l} \varepsilon_{i j}^{S F}(p) \varepsilon_{k l}^{S F}(q)-n_{i} \sigma_{i j}^{0}(p) \Omega_{j k}(\boldsymbol{n}) \sigma_{k l}^{0}(q) n_{l} & \boldsymbol{n} \neq 0
\end{array},\right.
$$

where $\sigma_{i j}^{0}(p)=C_{i j k l} \varepsilon_{k l}^{S F}(p)$, and $\Omega_{i j}^{-1}(\boldsymbol{n})=C_{i k l j} n_{k} n_{l}$. The fixed boundary condition (clapped boundary) used in the simulations of this paper corresponds to the state of a single grain embedded in a polycrystalline aggregate, which is constrained by the surrounding grains.

\subsection{Kinetic equation}

The following time-dependent Ginzburg-Landau (TDGL) equation (Gunton et al., 1983) is used to describe the temporal evolution of the structural order parameters during the MT

$$
\frac{d \eta_{p}(\boldsymbol{r}, t)}{d t}=-M \frac{\delta F}{\delta \eta_{p}(\boldsymbol{r}, t)}+\xi_{p}(\boldsymbol{r}, t), p=1 \sim 6
$$

where $M$ is a kinetic coefficient and $\xi_{p}(\boldsymbol{r}, t)$ is the Langevin noise term that meets the following fluctuation-dissipation theorem (Landau and Lifshitz, 1980; Wang and Khachaturyan, 1997):

$$
\left\langle\xi_{p}(\boldsymbol{r}, t) \xi_{p}\left(\boldsymbol{r}^{\prime}, t^{\prime}\right)\right\rangle=2 \frac{k_{B} T}{|\Delta f| l^{3}} \delta\left(\boldsymbol{r}-\boldsymbol{r}^{\prime}\right) \delta\left(t-t^{\prime}\right)
$$

where $k_{B}$ is the Boltzmann constant, $T$ is the absolute temperature, $|\Delta f|$ is the chemical driving force, $l$ is the length scale assigned to the computational grid increment, and $\delta$ is the delta function.

\subsection{Simulation results}

The phase field simulations are carried out by quenching the model system (a cubic cell with 
$64 \times 64 \times 64$ mesh points) down to $193 \mathrm{~K}$ with no external load. Periodic boundary conditions are applied in all three directions. To accelerate the nucleation process in the simulations, a relatively large amplitude of the Langevin noise term in Eq. (14) is first introduced during reduced time of 1, and then it is decreased by a factor of 10. Equation (14), with Eqs. (8) - (12) as inputs, is solved in the reciprocal space using a finite difference method. All the dimensionless materials parameters entering Eqs. (9), (10), (14), and (15) are listed in the Appendix A.

Figure 4 shows a typical example of microstructure evolution during MT, including nucleation and growth, and pattern formation, obtained from the phase field simulations. In Figs. 4(a)-(d), the austenite is set to be transparent and different variants of the martensite are plotted as isosurface with different colors. In Figs. 4(e)-(h), the austenite and martensites are represented by different colors. Figure 4(a) shows that single-variant martensitic particles of V3, V4 and V6 first appear. This is consistent with the prediction based on PTMC that a single variant can form an invariant habit plane with the parent phase. While V3, V4 and V6 continue to grow, new singlevariant particles of V2, V3, V4 and V5 (as indicated by the red arrows) nucleate (Fig. 4(b)) due to autocatalysis, followed by the formation of other single-variant particles. These single-variant particles grow, impinge upon each other (Figs. 4(c) and (d)) and eventually rearrange themselves into a characteristic polytwin domain structure (Figs. 4(e) - (h)) which corresponds to the C-II herringbone structure shown in Fig. 2(c). The volume fraction changes of the six martensitic variants during the MT are shown in Fig. 5. The nucleation and rapid growth of martensitic particles shown in Fig. 4 and 5 capture well the strong first-order nature of the MT. It is readily seen from Fig. 5 that before the total volume fraction of the martensitic phase reaches constants, the volume fractions of all individual variants increase. When the total volume fraction of the martensitic phase reaches a constant, coarsening begins and some of the variants (e.g., V3, V4, V5 and V6) grow at the expanse of some others (e.g., V1 and V2). Please note that this coarsening process is driven by both the reduction in elastic strain energy (i.e., strain-induced coarsening (Wang et al., 1992, 1991; Wen et al., 2002; Zhou et al., 2014)) and the reduction in domain wall energy, leading to the formation of a long-range ordered polytwin domain structure.

Because of the stochastic nature of the Langevin noise term, different patterns are generated in the phase field simulations. Figures 6(a)-(c) show three different types of twins obtained from the 
simulations (with different seeds for the random number generator), which are compound, type-I and type-II twins, in agreement with the predictions of the PTMC analysis presented in Section 2. In Fig. 6, the compound twin is formed by V3 and V4 and the type-I twin is formed by V3 and V5. Figure 6(c) shows more than one type of twins, including type-II twins formed by V3/V6 and V4/V5, and compound twins formed by V3/V4 and V5/V6. 


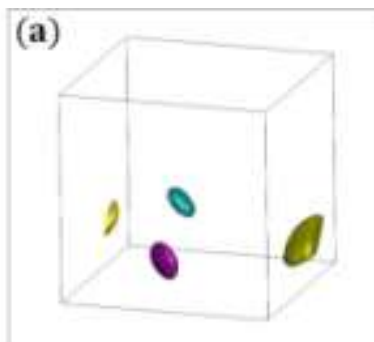

(e)

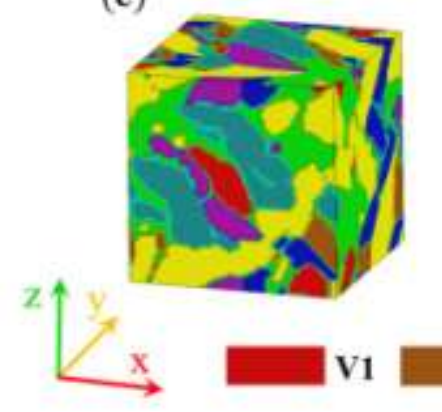

(b)

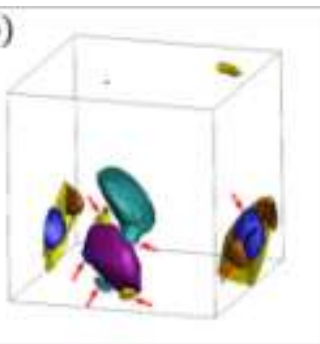

(f)

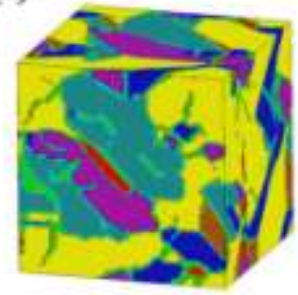

V2 (c)

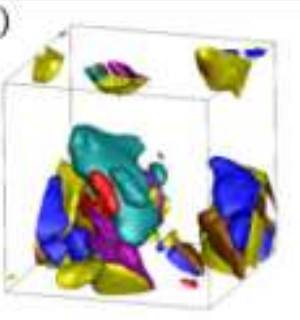

(g)

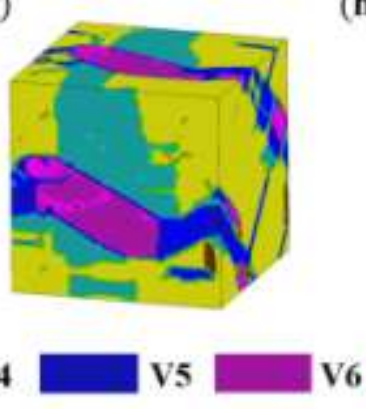

(d)

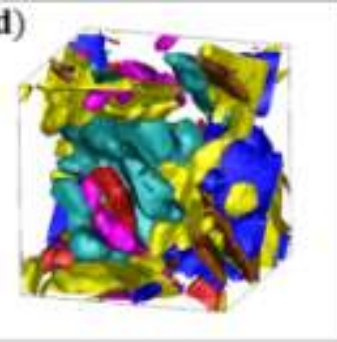

(h)

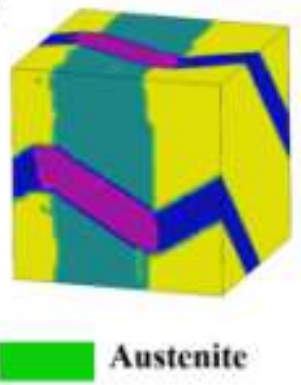

Fig. 4. Phase field simulation of microstructure evolution during MT in Ti2448 at 193K. (a)-(h) are microstructures at reduced time of 10.8, 15.6, 18.6, 21.6, 24, 33, 105.6, and 600, respectively. In order to show some morphological details of martensitic particles during their early stages of growth, the austenite is made transparent in (a) - (d). 


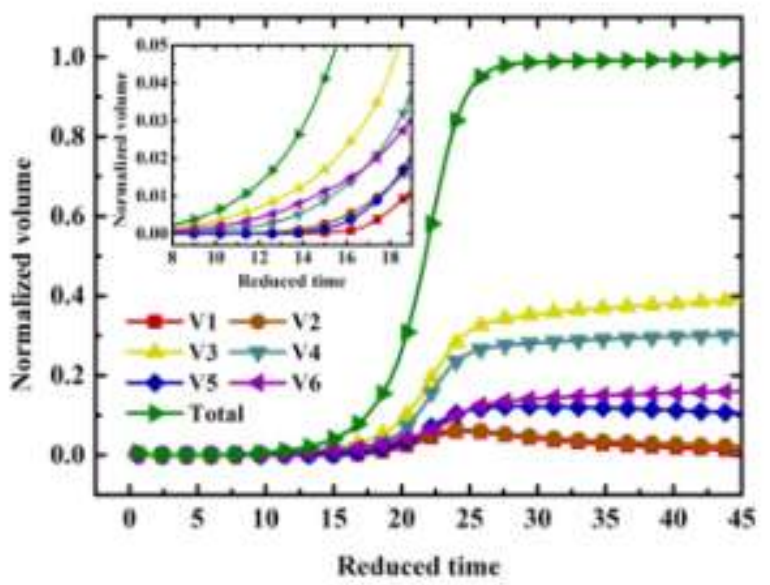

Fig. 5. Volume fraction changes of six martensitic variants during MT in Ti2448 at 193K. The inset shows volume fraction changes at the beginning of the transformation. 

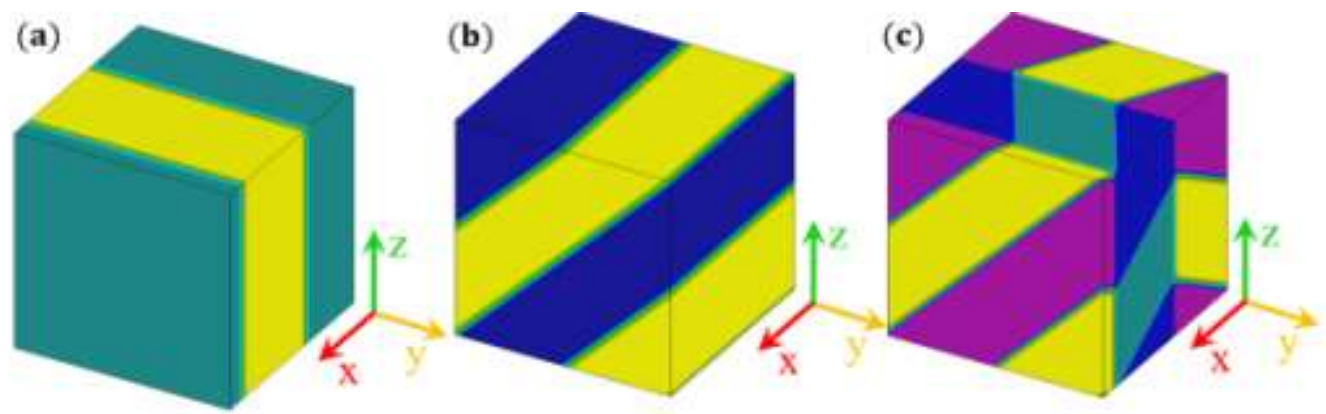

(d)

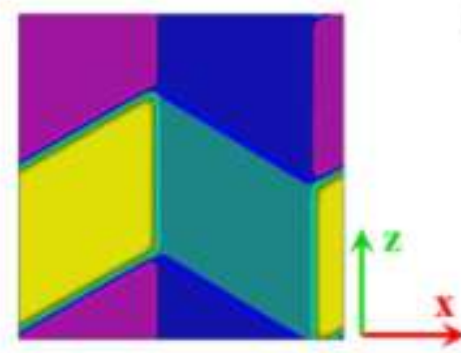

(e)

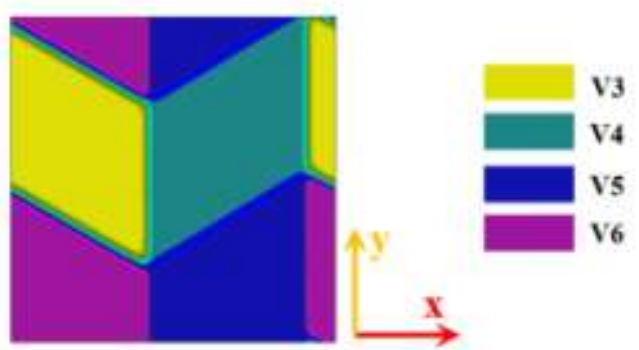

Fig. 6. Polytwinned microstructures predicted by the phase field simulations at $193 \mathrm{~K}$. (a) Compound twin, (b) type-I twin, (c) type-II twins. (d) and (e) are two dimensional projections of (c). 
The herringbone structure is a special kind of polytwinned martensitic domain structure. It has been observed frequently in both experiments and simulations (Gao et al., 2014; Jin et al., 2001; Otsuka and Ren, 2005; Wang and Khachaturyan, 2006). In Section 2.4, two kinds of herringbone structures, C-II and I-C, are predicted according to Eq. (5). Both of them are observed in the phase field simulations as well, as shown in Fig. 7. The C-II type herringbone structure (Fig. 7(a)) is formed by variants 1, 2, 3, and 4, and the I-C type (Fig. 7(e)) is formed by variants 1, 2, 5, and 6. In fact, the pattern shown in Fig. 6(c) is also a C-II herringbone structure formed by variants 3, 4, 5 and 6. These patterns are a subset of the solutions presented in Fig. 2.

\section{Experimental characterization}

To validate the twin structures predicted by the PTMC analysis and phase field simulations, an ingot of Ti2448 alloy with a diameter of $380 \mathrm{~mm}$ was fabricated via vacuum arc melting by using a Ti-Sn master alloy and pure $\mathrm{Ti}, \mathrm{Nb}$, and $\mathrm{Zr}$ as raw materials. The ingot was then hot-forged to a round bar of $55 \mathrm{~mm}$ in diameter at $1123 \mathrm{~K}$, followed by hot-rolling to $12 \mathrm{~mm}$ in diameter at 1073 $\mathrm{K}$. Then the material is cooled to room temperature in air, at which it matains as austenite. The samples are then loaded to a strain of $6 \%$ at room temperature in a uniaxial elongation test which is accompanied by MT before the TEM characterization. TEM foils were prepared by focused ion beam (FIB) milling in a Gatan 600 CTMP dual-beam system. Then high-resolution scanning transmission electron microscopy (HRSTEM) images were obtained with a FEI Titan 80-300 with Cs-correction on the electron probe, and operated at $300 \mathrm{kV}$. An example of the HRSTEM images of a typical twin structure is shown in Fig. 8, where the normal of the twin plane, $(01 \overline{1})_{\alpha}$, corresponds to the normal of the compound twin plane, $(010)_{\beta}$, formed by V1 and V2. This observation agrees well with the prediction of the PTMC analysis which is shown in Table B.1. 


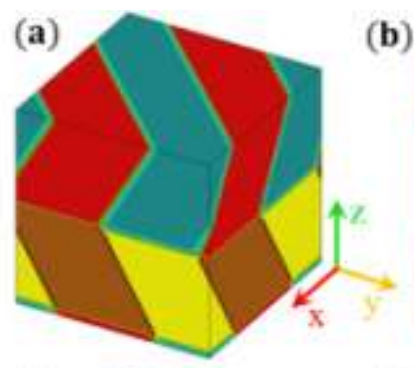

(b)

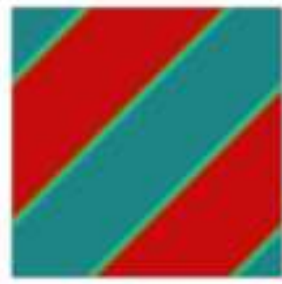

(c)

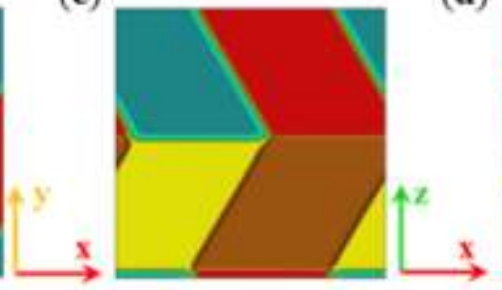

(d)

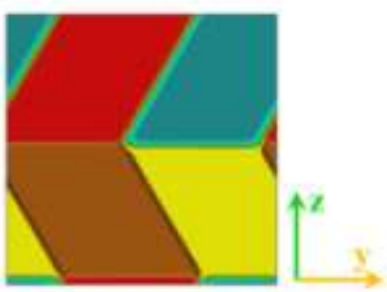

(e)

(f)
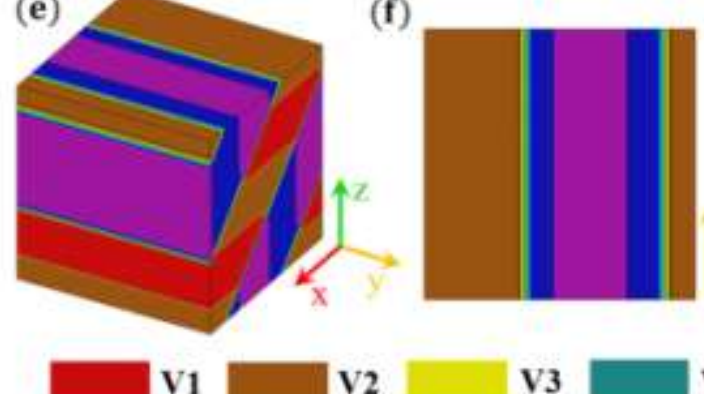

(g)
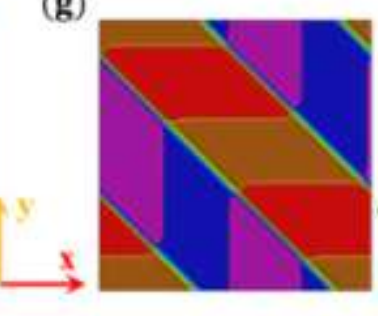

(h)

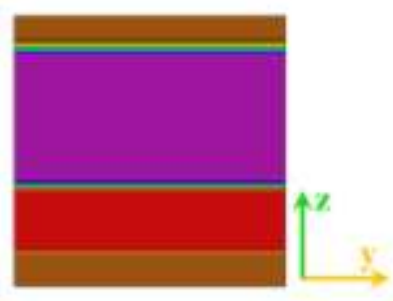

V4

\section{V5}

\section{V6}

Fig. 7. Phase field simulation prediction of two kinds of herringbone structures at $193 \mathrm{~K}$ : (a) C-II and (e) I-C. (b)-(d) and (f)-(h) are two dimensional projections of (a) and (e), respectively. 


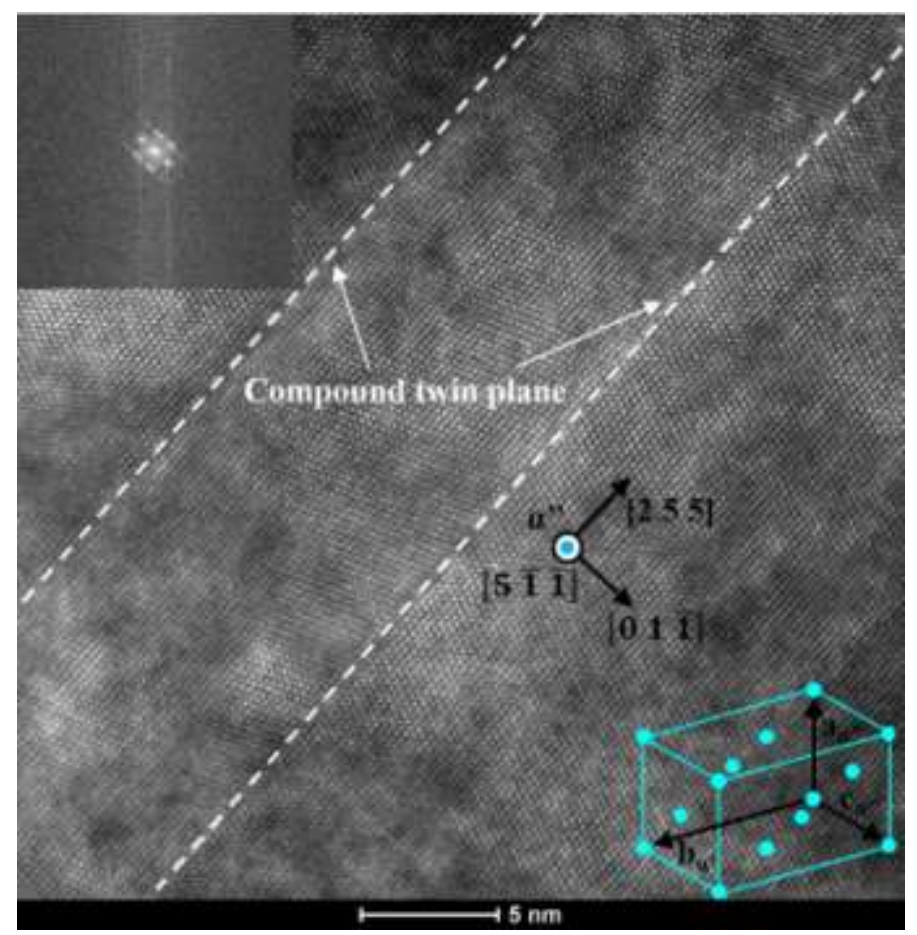

Fig. 8. HRSTEM image and diffraction pattern of a twin structure in Ti2448. Twin planes are marked with the dash lines, and the orientation of the TEM foil relative to the orientation of the crystal structure of the martensite is indicated by the insets. 


\section{Discussion}

5.1 Comparison of twin structures obtained via PTMC analysis, phase field simulation and scanning transmission electron microscopy (STEM) observation

As shown in Section 3.5, three types of twins, compound, type-I and type-II twins, are successfully reproduced by the phase field simulations. The twin planes predicted by the PTMC and those predicted by the phase field simulations (Fig. 6) are compared in Table 5, where it is found that the results obtained from the two different methods are almost identical. These predictions also agree with the twin structures of $\alpha$ " martensite observed in Ti2448 after $6 \%$ deformation (uniaxial elongation). For example, the typical compound twin structure formed by V1 and V2 is shown in Fig. 8, whose twin plane agrees well with the predictions by the PTMC analysis and phase field simulations.

Note that PTMC is a pure geometrical theory using the transformation matrix as inputs. The theory predicts twin plane and invariant plane normals. The KS theory, on the other hand, is based on elastic energy minimization. As inputs, both eigenstrains and elastic constants are required (the eigenstrains can be calculated from the transformation matrix) and, as output, the twin plane and habit plane normals can be predicted. In the current study, the elastic constants and the transformation strains from experimental measurements (Liu et al., 2013) are used. Also note that PTMC analysis and phase field simulation should yield exactly the same results if invariant plane exist between two adjacent domains (i.e., the habit plane is an invariant plane). This is the reason why we obtained almost identical results in Table 5. However, predictions of PTMC could be different from that of phase field simulation when local elastic deformation becomes unavoidable in certain cases (Gao et al., 2014).

Table 5 Comparison of twin planes getting from PTMC and phase field simulation

\begin{tabular}{cccc}
\hline Twin pairs & Twinning type & PTMC & Phase field simulation \\
\hline V3\&V4 & Compound & $(-1,0,0)$ & $(-1,0,0)$ \\
V5\&V6 & Compound & $(1,0,0)$ & $(1,0,0)$ \\
V3\&V5 & Type-I & $(0,1,-1)$ & $(0,1,-1)$ \\
V3\&V6 & Type-II & $(0.3911,0.6508,-0.6508)$ & $(0.3914,0.6513,-0.6501)$ \\
\hline
\end{tabular}




\subsection{Geometrical Compatibility}

The above PTMC analysis and phase field simulations demonstrate the plethora of building blocks of martensite that provide a strong ability for self-accommodation as well as for adaptation to existing defects without the necessity to generate new dislocations. In addition to the rich variety of the self-accommodating modes of martensite, the excellent geometric compatibilities of the martensite with the parent phase are also the main reasons behind the lack of new dislocations generated during the MT and good fatigue resistance of Ti2448 (S. Q. Zhang et al., 2011). Figure 9 shows the shear stress fields induced by a single variant martensitic particle having a size of $\sim 60 \mathrm{~nm}$ (Fig. 9(a)) in Ti2448 (Fig. 9(b)) and Ti-49.8Ni (Fig. 9(c)). It is readily seen that the MT in Ti2448 has much lower local stresses and thus much lower probability to activate dislocations as compared to that in the conventional SMAs such as the TiNi based alloys.

A good geometrical compatibility, especially when the cofactor conditions (James et al., 2005) are satisfied, has been shown to exhibit enhanced reversibility and functional fatigue resistance (Song et al., 2013). The cofactor conditions are

$$
\begin{gathered}
\lambda_{2}=1 \\
\mathrm{CCI}=\operatorname{tr} \boldsymbol{U}^{2}-\operatorname{det} \boldsymbol{U}^{2}-\frac{|\boldsymbol{a}|^{2}|\boldsymbol{n}|^{2}}{4}-2 \geq 0 \\
\mathrm{CCII}=\mathbf{a} \cdot \boldsymbol{U} \operatorname{cof}\left(\boldsymbol{U}^{2}-\boldsymbol{I}\right) \boldsymbol{n}=0
\end{gathered}
$$

where $\lambda_{2}$ is the middle eigenvalue of $\boldsymbol{U}, \boldsymbol{a}, \boldsymbol{n}$ and $\boldsymbol{U}$ have the same meaning as those in Eq. (3), $\operatorname{tr} \boldsymbol{U}^{2}$ and $\operatorname{det} \boldsymbol{U}^{2}$ denotes the trace and determinant of $\boldsymbol{U}^{2}$, respectively, $\operatorname{cof}\left(\boldsymbol{U}^{2}-\boldsymbol{I}\right)$ is the cofactor matrix of $\boldsymbol{U}^{2}-\boldsymbol{I}$. As examples for Ti2448, the value of $\lambda_{2}$, CCI, and CCII of the type I twin formed by variant 1 and 3 are $1.003,-8.843 \times 10^{-4}, 3.537 \times 10^{-5}$, respectively. For the type II twin these values are $1.003,-9.355 \times 10^{-4}$ and $3.415 \times 10^{-5}$, respectively, and for the compound twin these values are 1.003, $1.3 \times 10^{-3}$ and $-4.272 \times 10^{-5}$, respectively. These values, even though not satisfy exactly Eq. (16)-(18), are close to those of $\mathrm{Cu}_{68} \mathrm{Zn}_{15} \mathrm{Al}_{17}$ that has 
the highest compatibility in the CuZnAl SMA system (James et al., 2005). For the type I twin formed by variant 1 and 12 in $\mathrm{Cu}_{68} \mathrm{Zn}_{15} \mathrm{Al}_{17}$, for example, the value of $\lambda_{2}, \mathrm{CCI}$, and CCII are $1.0026,-3.04 \times 10^{-2}$ and $4.2719 \times 10^{-4}$, respectively.

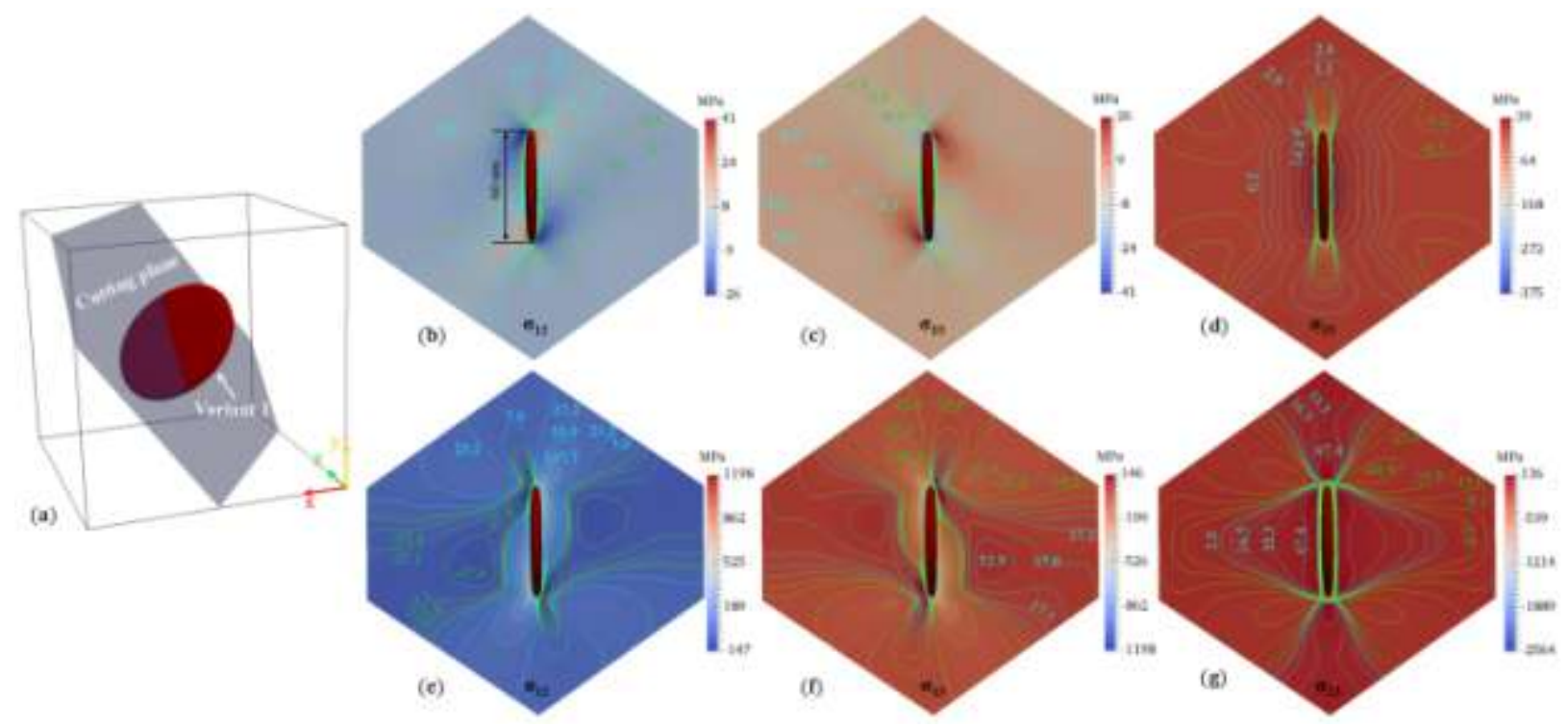

Fig. 9 Two dimensional sections of three dimensional shear stress fields, $\sigma_{12}, \sigma_{13}, \sigma_{23}$, associated with (a) a single variant martensitic particle of around 60nm in (b)-(d) Ti2448 and (e)-(g) Ti$49.8 \mathrm{Ni}$. 
The stress fields of the type I and type II twins as well as that of the herringbone structure are shown in Fig. 10 (a)-(c), respectively. For comparison, the stress fields of the type I and type II twins and the herringbone structure in Ti-49.8Ni are also shown in Fig. 10(d)-(f). The configurations of type I and type II twins and the herringbone structure of Ti2448 and those of Ti-49.8Ni are shown in Fig. 10 (g)-(i) and Fig. 10 (j)-(l), respectively. They are obtained by relaxing internally twinned martensitic particles that are placed in the center of the phase field computational cell. The initial configurations of the internally twinned martensitic particles are designed following their habit plane, twin plane and the ratio of volume fractions between the variants predicted by PTMC. The austenite is set to be transparent and the different martensitic variants are represented by different colors in Fig. 10 (g)-(l). It is readily seen that the internal stresses generated by the martensitic domains in Ti2448 are much lower than those generated in Ti-49.8Ni. Thus the excellent geometrical compatibility of the martensitic domains in Ti2448 minimizes stress concentration during the MT and limits conventional dislocation activities. 

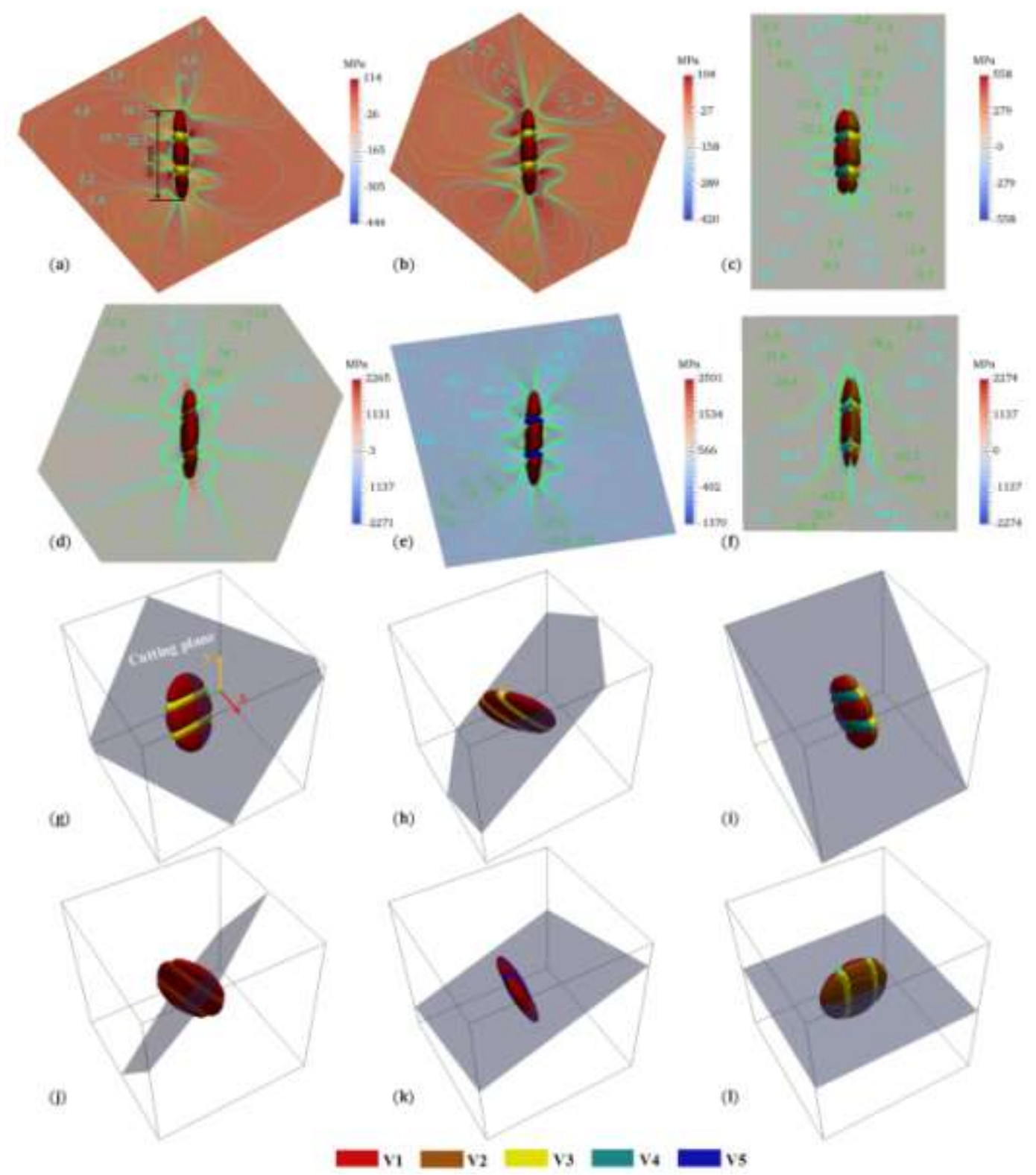

Fig. 10 (a)-(c) are shear stress fields, $\sigma_{13}$, induced by (g) type I twin, (h) type II twin, and (i) C-II herringbone structure in Ti2448, respectively. (d)-(f) are shear stress fields corresponding to (j) type I twin, (k) type II twin, and (1) C-II herringbone structure in Ti-49.8Ni, respectively. 


\subsection{Herringbone structure}

Herringbone structures play an essential role in accommodating deformations. For example, during MT, they are building blocks of self-accommodating patterns; during shape memory application, they are the main deformation carrier in responds to mechanical loading. Below we analyze the responds of C-II and I-C herringbone structures to external load.

The normal of twin planes shown in Fig. 7 are available in both Fig. 2 and Appendix B. Comparing Figs. 7(c) and (g), and Fig. 6(e) with Figs. 2(a), (e), and (c), respectively, it is found that the results obtained from the phase field simulations agree well with the results obtained from the PTMC analyses. Figure 7 indicates that it is reasonable to assume that each variant takes up a quarter of the total volume in a herringbone structure under the fixed boundary condition used in this simulation. Then the average strains of the herringbone structures shown in Figs. 7(a) and (e) can be calculated via Eq. (19) and Eq. (20), respectively,

$$
\begin{aligned}
\frac{1}{4}\left[\varepsilon_{i j}^{S F}(1)+\varepsilon_{i j}^{S F}(2)+\varepsilon_{i j}^{S F}(3)+\varepsilon_{i j}^{S F}(4)\right] & =0.001\left[\begin{array}{ccc}
-4.51 & 0 & 0 \\
0 & -4.51 & 0 \\
0 & 0 & 13.75
\end{array}\right], \\
\frac{1}{4}\left[\varepsilon_{i j}^{S F}(1)+\varepsilon_{i j}^{S F}(2)+\varepsilon_{i j}^{S F}(5)+\varepsilon_{i j}^{S F}(6)\right] & =0.001\left[\begin{array}{ccc}
-4.51 & 0 & 0 \\
0 & 13.75 & 0 \\
0 & 0 & -4.51
\end{array}\right] .
\end{aligned}
$$

Similarly, the average strain of a herringbone structure consisting of variants $3,4,5$, and 6 can be calculated as

$$
\frac{1}{4}\left[\varepsilon_{i j}^{S F}(3)+\varepsilon_{i j}^{S F}(4)+\varepsilon_{i j}^{S F}(5)+\varepsilon_{i j}^{S F}(6)\right]=0.001\left[\begin{array}{ccc}
13.75 & 0 & 0 \\
0 & -4.51 & 0 \\
0 & 0 & -4.51
\end{array}\right] .
$$

Equations (19)-(21) demonstrate that the principal axes of the averaged strains of C-II and I-C herringbone structures coincide with each other and run parallel to the reference coordinate system when the four variants have the same volume fraction. Furthermore, variant combinations of (V1, V2, V3, V4), (V1, V2, V5, V6) and (V3, V4, V5, V6) are more efficient in accommodating strains along the $z, y$, and $x$ directions, respectively, as compared to the other directions. 
In terms of the average strain, there is no difference in the transformation strain accommodation between the C-II and I-C types of herringbone structures when the variants in the structures have the same volume fraction. However, this is not the case anymore when the volume fractions of different variants are not identical.

In the C-II herringbone structure there are two types of twins, Compound and Type II, as schematically illustrated in Fig. 11(a). Variants in the compound twin have to have the same volume fraction in order to maintain the twin boundary compatibility, while variants in the typeII twin do not have such a constraint. The volume ratio of variants residing on two sides of the type-II twin plane would vary according to the external boundary condition and applied stress (Gao et al., 2014) as well as other confinements. The variation of volume ratio of variants could happen in the I-C herringbone structure as well, as shown schematically in Fig. 11(b). As a result, part of V2 and V3 will transform to V1 and V4, respectively, during de-twinning under applied stress/strain, and the volume fraction of the four variants will not be the same any more. Such a variant-variant transformation should also influence the ability of the system to accommodate deformations. The volume fractions of variants can be measured via their edge length as shown in Fig. 11. The average strain (decomposed into spherical and deviatoric strain tensor) of the C-II and I-C herringbone structures are then obtained via

$$
\begin{aligned}
& \frac{1}{2}\left[r \varepsilon_{i j}^{S F}(1)+r \varepsilon_{i j}^{S F}(2)+(1-r) \varepsilon_{i j}^{S F}(3)+(1-r) \varepsilon_{i j}^{S F}(4)\right]= \\
& 0.001\left[\begin{array}{ccc}
1.57 & 0 & 0 \\
0 & 1.57 & 0 \\
0 & 0 & 1.57
\end{array}\right]+0.001\left[\begin{array}{ccc}
12.18-36.53 r & 0 & 0 \\
0 & -24.35+36.53 r & 0 \\
0 & 0 & 12.18
\end{array}\right] \text {, } \\
& \frac{1}{2}\left[r \varepsilon_{i j}^{S F}(1)+(1-r) \varepsilon_{i j}^{S F}(2)+(1-r) \varepsilon_{i j}^{S F}(3)+r \varepsilon_{i j}^{S F}(4)\right]= \\
& 0.001\left[\begin{array}{ccc}
1.57 & 0 & 0 \\
0 & 1.57 & 0 \\
0 & 0 & 1.57
\end{array}\right]+0.001\left[\begin{array}{ccc}
-6.09 & 0 & -5.49+10.98 r \\
0 & -6.09 & 5.49-10.98 r \\
-5.49+10.98 r & 5.49-10.98 r & 12.18
\end{array}\right] \text {. }
\end{aligned}
$$



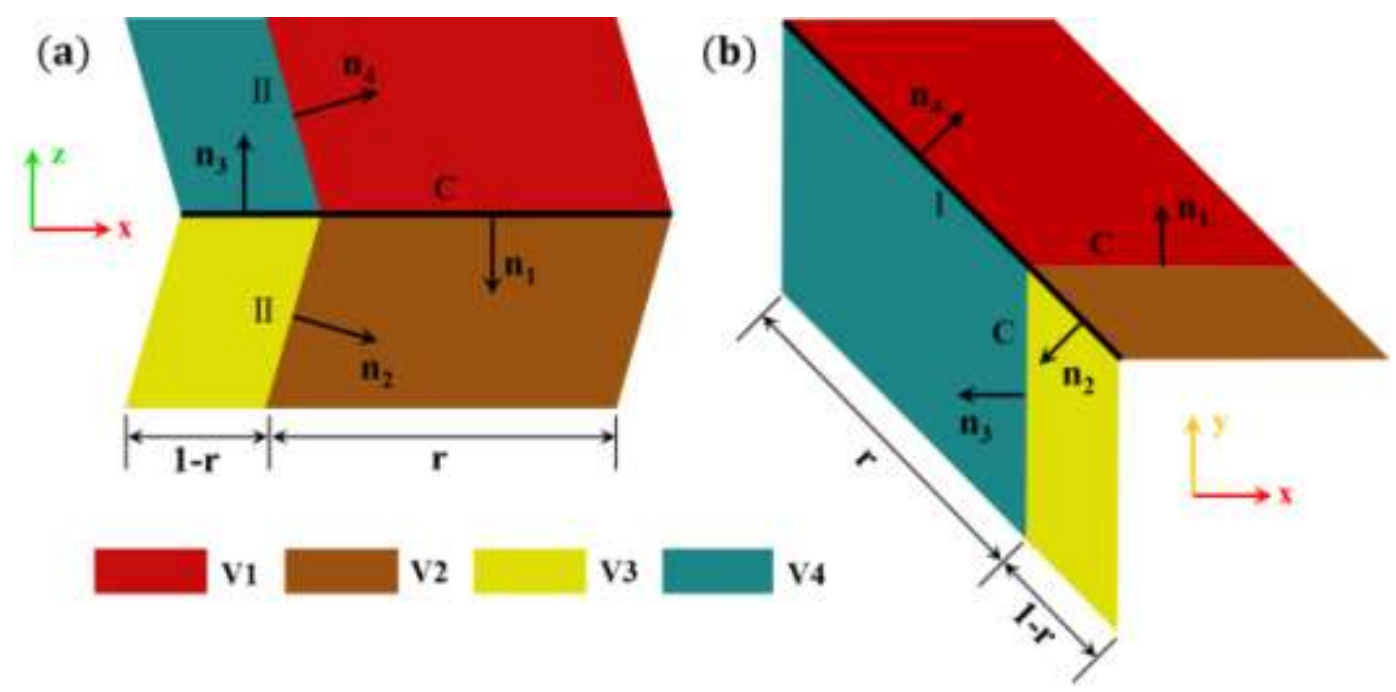

Fig. 11 Schematic drawings of (a) C-II and (b) I-C herringbone structures consisting of variants with non-equal volume fractions. $r$ denotes the volume fraction of a variant in a herringbone structure, which can be calculated via the edge length of variants as shown in (a) and (b). 
Equation (22) and (23) have the same spherical strain tensor, which indicates that volume strains associated with the C-II and I-C herringbone structures are the same. But Eq. (22) involves only the diagonal components no matter what the value of $r$ is, which indicates that the principal strain axes of the C-II herringbone structure, regardless of the volume ratio between different variants, always parallel to the three cube axes of the parent phase (the reference coordinate system). However, Eq. (23) shows that the principal strain axes of the I-C herringbone structure do not parallel to the reference coordinate system unless $r=0.5$, which corresponds to the case shown in Eq. (19) where all four variants have the same volume fraction.

Figure 12(a) shows the variation of strain states (by the two red arrows) of the C-II herringbone structure with respect to $r$ in the principle strain space. We can readily see that the principle strain axes of the C-II herringbone structures do not depend on $r$ (Fig. 12(a)). The two red arrows and all the solid dots in Figure 12(a) indicate the deformation states that a C-II herringbone structure could accommodate by varying $r$ from 0 to 1 . External loads that yield deformation states coincide with or close to those shown in Fig. 12(a) will prefer the C-II herringbone structure. Considering the fixed principal strain axes of the C-II herringbone structures, such kind of external loads could be uni-, bi- and tri-axial tension and compression.

The rotation of the principle strain axes of the $\mathrm{I}-\mathrm{C}$ herringbone structure with respect to $r$ is shown in Fig. 12(b). In contrast to the C-II herringbone structure, the principle strain axes of the I-C herringbone structure rotates continuously from the cyan to the blue and then to the purple ones as $r$ increases from 0 to 0.5 to 1 , which means that the $\mathrm{I}-\mathrm{C}$ herringbone structure could accommodate deformations whose principle strain directions vary. External loads that induce this kind of deformation modes include torsion and the combination of tension and torsion.

In fact, Eqs. (22) and (23) also provide some insight into the experimental observation that the I$\mathrm{C}$ and $\mathrm{C}$-II herringbone structures do not usually appear with equal probability (Otsuka and Ren, 2005). For example, in $\mathrm{Ni}-\mathrm{Ti}$ and $\mathrm{Cu}-\mathrm{Al}-\mathrm{Ni}$ systems, it is observed that the $\mathrm{C}$-II structure with type-II twinning is dominant over the I-C with type-I twinning, even though they are conjugate twinning mode from the crystallography. However, PTMC is a geometrical theory based on Cauchy-Born hypothesis (Bhattacharya, 2003), and crystal lattice is treated as continuum under 
this framework. As a result, lattice discreteness and anisotropy in crystal properties (such as elastic modulus) cannot be considered. In order to understand the formation preference of I-C and C-II structures, factors related to crystal anisotropy and lattice discreteness have to be taken into account. For example, considering a uniaxial tension applied to a sample, the C-II structure will be favored if the loading direction is along $\langle 100\rangle_{\beta}$, while the I-C structure will be favored if the loading direction is along $\langle 110\rangle_{\beta}$. Similarly, elastic anisotropy may also have an effect on the formation preference of these two types of herringbone structures.

Defects have been utilized widely to alter martensitic transformation pathways and domain structures in ferroelastic systems. In particular, defect engineering has been shown to alter effectively the transformation pathway from normal MT to strain glass transition and produce single-variant nano-sized martensitic domains wihtout long-range order in systems including NiTi (Ji et al., 2013; Sarkar et al., 2005; Wang et al., 2010; Zhou et al., 2010), Gum metals (Y. Wang et al., 2014), Ni-Mn (Entel et al., 2014) and AuCuAl (Liu et al., 2011). According to (Wang et al., 2016), the SFTS of a MT has a strong impact on the possibilty to achieve a srain glass state of a ferroelastic system. Since the SFTS of the $\alpha$ " martensite in Ti2448 has relatively small values, it is expected that a strain glass state can be easily achieved in this system, which will be the subject of a follow-up paper. 

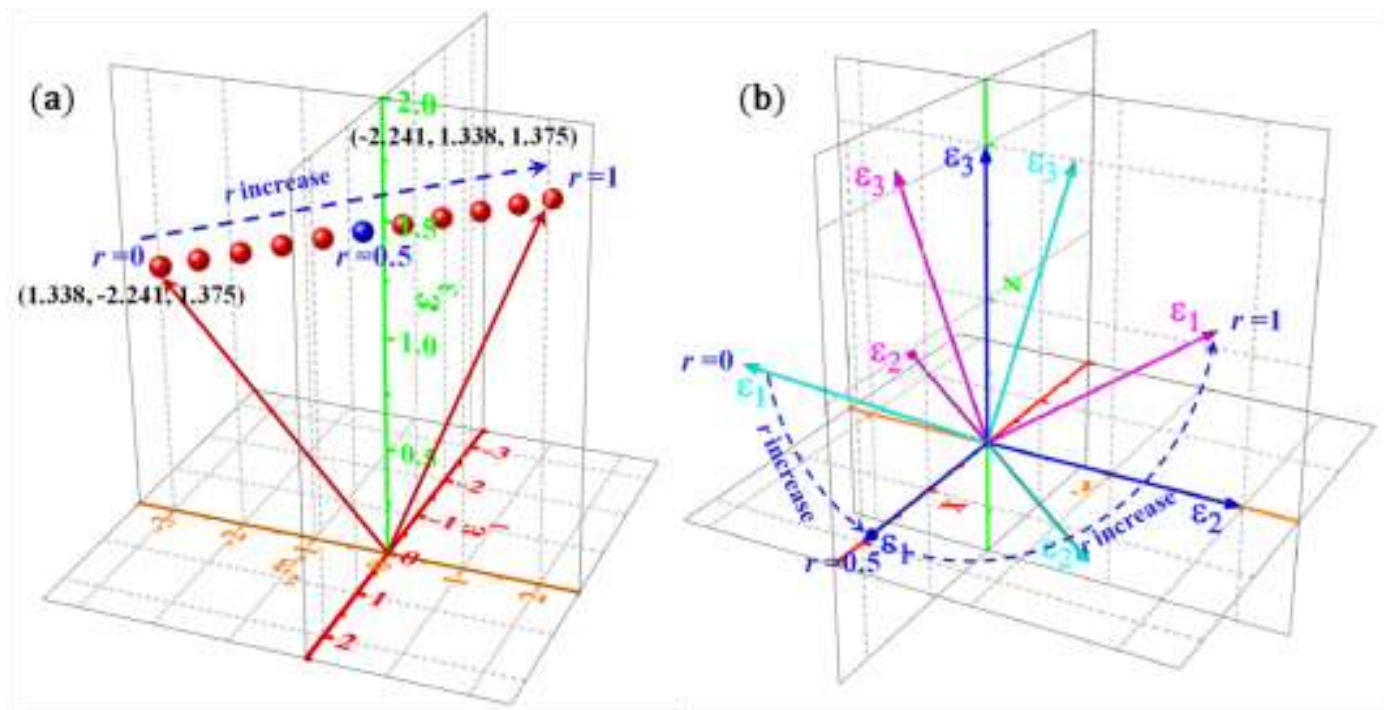

Fig. 12 (a) the variation of strain state (indicated by the red arrows) of the C-II herringbone structure consisting of V1, V2, V3 and V4 in principle strain space and (b) the rotation of the principle strain axes of the I-C herringbone structure consists of V1, V2, V3 and V4 with respect to volume fraction $r$. The two red arrows in (a) indicate the strain states of the C-II herringbone structure corresponding to $r=0$ and $r=1$, respectively. The cyan, blue and purple sets of principle strain axes correspond to $\mathrm{r}=0,0.5$, and 1 , respectively. The blue solid dot in (a) correspond to the case of $r=0.5$. 


\subsection{Limitations of the phase field model}

The phase field model employed in the current study has several limitations, which are, in fact, reasonable simplifications for the problem at hands. First of all, the small strain formulation is used in calculating the elastic energy induced by the lattice misfit between the martensite and parent phase and among different variants of the martensite. This is because no large elastic deformation is expected during the MT according to the crystallographic analysis. The stress-free transformation strain is small (Hao et al., 2012) and ample building blocks are available for the formation of various self-accommodated structures during the MT, which renders large elastic deformations unnecessary.

Secondly, the martensitic phase is assumed to have the same elastic constants as that of the parent phase because of the lack of consistent experimental data. Several reports claim that the Young's modulus of the parent $\beta$ phase was higher than that of the $\alpha$ " martensitic phase (Fedotov, 1973; Ho et al., 1999; Matsumoto et al., 2005), but the opposite result is also reported (Graft and Rostoker, 1957). Hao et al. (Hao et al., 2002) argued that the Young's modulus of the $\beta$ phase may be lower or higher than that of the $\alpha$ " phase depending on the alloy composition. Fortunately, it has been proved that this assumption does not prevent the phase field simulations from reproducing the major features of martensitic structures (Wang and Khachaturyan, 1997). Nevertheless, when more consistent modulus data become available, inhomogeneous modulus microelasticity approaches (Wang et al., 2002; Zhou et al., 2008) should be used.

Thirdly, the inertial effect (Lookman et al., 2003), which is important when the long wave oscillation are concerned, is ignored in the present model because the main focus of the work is on self-accommodated pattern formation during the MT rather than its kinetics.

Fourthly, the model is formulated for perfect crystals and does not take into account the effects of structural non-uniformities associated with extended defects and composition non-uniformities. These non-uniformities could alter the pathway of the MT and hence change the domain structures formed (Das and Tarafder, 2009; Hao et al., 2016; Lovey et al., 2004; Zhang et al., 2015). The effects of these spatial non-uniformities on the MT pathways in this particular system will be investigated in a separate paper. 
Fifthly, the six martensitic variants represented by a set of structural order parameters are equivalent in terms of their Bravais lattice and the only difference among them is their orientation. Thus the interfacial energy between the austenite and these martensitic variants as well as that between different martensitic variants are equal. In the current model, we didn't distinguish twin boundaries for different kinds of twins because of the lack of experimental data. In other words, the boundary energies are equal for all the types of twin boundaries. The consideration of boundary energy anisotropy in phase field method is straight forward (see, e.g., Ma et al., 2004 and Ma et al., 2006) when experimental data become available.

Finally, the temperature dependence of the lattice parameters and elastic constants are not considered in the present model. The linear thermal expansition coefficient of Ti2448 is in the order of $10^{-5} / \mathrm{K}$ (Hao et al., 2016), and the transformation strain is in the order of $10^{-2}$ (Otsuka and Ren, 2005). Therefore, the effect of temperature on the transformation strains is negligible. The elastic constants are usually weak function of temperature in a temperature range far below the melting temperature (Anderson, 1966; Collings, 1986). Thus elastic constants measured at room temperature (Liu et al., 2013) are used in our calculations. Experimental studies reveal that the Young's modulus of Ti-Nb binary alloys is indeed a weak function of temperature. Taking Ti-36at.\% Nb as an exanple, its Young's modulus is $82 \mathrm{GPa}$ at $300 \mathrm{~K}$ and $83 \mathrm{GPa}$ at $4 \mathrm{~K}$ (Collings, 1986). This indicates that the Young's modulus changs only about $3.4 \times 10^{-3} \mathrm{GPa}$ per Kelvin within this temperature range of interest, which is negligible as compared to the Young's modulus itself ( $~ 80 \mathrm{GPa})$. Moreover, because of the diverse strain accommodating modes we have shown in this study, especially the excellent geometrical compatibilities of the twins and herringbone structures, elastic distortions in the self-accommodated martensitic domain patterns are expected to be small and thus the effect from the temperature-dependent part of the elastic modulus is small as well.

\section{Summary}

Both crystallographic analysis and phase field simulations have revealed many unique features of microstructure evolution during the cubic to orthorhombic martensitic transformation (MT) in 
Ti2448. First of all, in the crystallographic analysis based on the phenomenological theory of martensite crystallography (PTMC) we find 30 twin planes, including 6 compound types, 12 type-I and 12 type-II. The formations of all three types of twins have been observed in the phase field simulations based on Landau theory (Landau and Lifshitz, 1980) and KhachaturyanShatalov's microelasticity theory (KS theory) (Khachaturyan, 1983) of MTs. Secondly, the crystallographic analysis also predicts 12 single variant habit planes in addition to the 96 internally twinned habit planes based on the fact that the stress-free transformation strain (SFTS) of a single variant is an invariant plane strain in this system. The nucleation, growth and twinning and de-twinning of martensitic variants are analyzed in details based on the phase field simulation results. Thirdly, two kinds of herringbone structures, C-II and I-C, are captured by the phase field simulations, which agree well with both experimental observations and PTMC predictions. The C-II herringbone structure could accommodate deformations whose principle strain directions are fixed such as uni-, bi-, and tri-axial tension and compression, while the I-C herringbone structure could accommodate deformations that have variable principle strain directions such as torsion and combination of torsion and tension or compression. Finally, the geometrical compatibilities of the twin and herringbone structures formed in Ti2448 are found similar to those of the twins formed in $\mathrm{Cu}_{68} \mathrm{Zn}_{15} \mathrm{Al}_{17}$, which have the highest compatibility in the CuZnAl SMA system (James et al., 2005), in terms of their fulfillments of the co-factor conditions. As lattice mismatch between the parent and martensitic phases plays a critical role on the elastic and plastic deformation at interfaces, the excellent geometrical compatibility in Ti2448 renders the local stresses associated with the martensitic domain structures to be approximately one order of magnitude smaller than those found in the NiTi-based commercial SMAs, without considering possible differences in elastic properties austenitic and martensitic phases in these alloys.

The ample building blocks and their excellent geometrical compatibilities for fully selfaccommodated strain domain structures found in Ti2448 in this study provide the system with great flexibility to accommodate arbitrary defect structures and deformation states, which may have contributed to its unprecedented mechanical properties including ultralow apparent elastic modulus, high strength, good fatigue resistance and super-elasticity in a broad temperature range. 


\section{Acknowledgement}

JM Zhu and $\mathrm{HH}$ Wu acknowledge the financial supports from the Joint School of XJTU and HKUST and from the Hong Kong Research Grants Council under the General Research Fund (Project number, 622911); YG and YW acknowledge the financial supports of the US Natural Science Foundation Grant No. DMR-1410322 and the US Department of Energy Grant No. DESC0001258; TY Zhang acknowledges the financial support of the Science and Technology Commission of Shanghai Municipality Grant No.14DZ2261200.

\section{Reference}

Anderson, O.L., 1966. Derivation of Wachtman's Equation for the Temperature Dependence of Elastic Moduli of Oxide Compounds. Phys. Rev. 144, 553-557. doi:10.1103/PhysRev.144.553

Artemev, A., Wang, Y., Khachaturyan, A.G., 2000. Three-dimensional phase field model and simulation of martensitic transformation in multilayer systems under applied stresses. Acta Mater. 48, 2503-2518. doi:10.1016/S1359-6454(00)00071-9

Bhattacharya, K., 2003. Microstructure of Martensite: Why It Forms and How It Gives Rise to the Shape-Memory Effect. Oxford University Press, Oxford.

Bowers, M.L., Chen, X., De Graef, M., Anderson, P.M., Mills, M.J., 2014. Characterization and modeling of defects generated in pseudoelastically deformed NiTi microcrystals. Scr. Mater. 78-79, 69-72. doi:10.1016/j.scriptamat.2014.02.001

Bowles, J.S., Mackenzie, J.K., 1954. The crystallography of martensite transformations I. Acta Metall. 2, 129-137. doi:10.1016/0001-6160(54)90102-9

Chen, L.-Q., 2002. Phase-Field Models for Microstructure Evolution. Annu. Rev. Mater. Res. 32, 113-140. doi:10.1146/annurev.matsci.32.112001.132041

Chen, X., Srivastava, V., Dabade, V., James, R.D., 2013. Study of the cofactor conditions: Conditions of supercompatibility between phases. J. Mech. Phys. Solids 61, 2566-2587. doi:10.1016/j.jmps.2013.08.004

Collings, E.W., 1986. Applied Superconductivity, Metallurgy, and Physics of Titanium Alloys. Springer US, Boston, MA. doi:10.1007/978-1-4613-2095-1

Cui, J.P., Hao, Y.L., Li, S.J., Sui, M.L., Li, D.X., Yang, R., 2009. Reversible movement of homogenously nucleated dislocations in a $\beta$-titanium alloy. Phys. Rev. Lett. 102, 1-4. doi:10.1103/PhysRevLett.102.045503

Das, A., Tarafder, S., 2009. Experimental investigation on martensitic transformation and fracture morphologies of austenitic stainless steel. Int. J. Plast. 25, 2222-2247. 
doi:10.1016/j.ijplas.2009.03.003

Delville, R., Malard, B., Pilch, J., Sittner, P., Schryvers, D., 2011. Transmission electron microscopy investigation of dislocation slip during superelastic cycling of Ni-Ti wires. Int. J. Plast. 27, 282-297. doi:10.1016/j.ijplas.2010.05.005

Eggeler, G., Hornbogen, E., Yawny, A., Heckmann, A., Wagner, M., 2004. Structural and functional fatigue of NiTi shape memory alloys. Mater. Sci. Eng. A 378, 24-33. doi:10.1016/j.msea.2003.10.327

Entel, P., Gruner, M.E., Comtesse, D., Sokolovskiy, V. V., Buchelnikov, V.D., 2014. Interacting magnetic cluster-spin glasses and strain glasses in Ni-Mn based Heusler structured intermetallics. Phys. status solidi 251, 2135-2148. doi:10.1002/pssb.201451059

Fedotov, S.G., 1973. Titanium Science and Technology. Plenum Press, Boston.

Finel, A., Le Bouar, Y., Gaubert, A., Salman, U., 2010. Phase field methods: Microstructures, mechanical properties and complexity. Comptes Rendus Phys. 11, 245-256. doi:10.1016/j.crhy.2010.07.014

Gao, Y., Shi, R., Nie, J.-F.F., Dregia, S.A., Wang, Y., 2016. Group theory description of transformation pathway degeneracy in structural phase transformations. Acta Mater. 109, 353-363. doi:10.1016/j.actamat.2016.01.027

Gao, Y., Zhou, N., Wang, D., Wang, Y., 2014. Pattern formation during cubic to orthorhombic martensitic transformations in shape memory alloys. Acta Mater. 68, 93-105. doi:10.1016/j.actamat.2014.01.012

Geetha, M., Singh, A.K., Asokamani, R., Gogia, A.K., 2009. Ti based biomaterials, the ultimate choice for orthopaedic implants - A review. Prog. Mater. Sci. 54, 397-425. doi:10.1016/j.pmatsci.2008.06.004

Graft, W., Rostoker, W., 1957. The Measurement of Elastic Modulus of Titanium Alloys, in: Symposium on Titanium: The Second Pacific Area National Meeting. ASTM, West Conshohocken, PA, p. 130.

Gunton, J.D., San Miguel, M., Sahni, P.S., 1983. The Dynamics of First Order Phase Transitions, in: Domb, C., Lebowitz, J.L. (Eds.), Phase Transitions and Critical Phenomena. Academic Press, New York, pp. 267-466.

Guo, Z., Fu, J., Zhang, Y.Q., Hu, Y.Y., Wu, Z.G., Shi, L., Sha, M., Li, S.J., Hao, Y.L., Yang, R., 2009. Early effect of Ti-24Nb-4Zr-7.9Sn intramedullary nails on fractured bone. Mater. Sci. Eng. C 29, 963-968. doi:10.1016/j.msec.2008.08.019

Gurtin, M.E., 1983. Two-phase deformations of elastic solids. Arch. Ration. Mech. Anal. 84, 129. doi:10.1007/BF00251547

Hahn, T., 2006. Graphical symbols for symmetry elements in one, two and three dimensions. Int. Tables Crystallogr. Vol. A Space-gr. symmetry 2, 7-11. doi:10.1107/97809553602060000503

Hao, Y.L., Li, S.J., Prima, F., Yang, R., 2012. Controlling reversible martensitic transformation in titanium alloys with high strength and low elastic modulus. Scr. Mater. 67, 487-490. 
doi:10.1016/j.scriptamat.2012.06.011

Hao, Y.L., Li, S.J., Sun, B.B., Sui, M.L., Yang, R., 2007. Ductile titanium alloy with low poisson's ratio. Phys. Rev. Lett. 98, 1-4. doi:10.1103/PhysRevLett.98.216405

Hao, Y.L., Li, S.J., Sun, S.Y., Yang, R., 2006. Effect of Zr and Sn on Young's modulus and superelasticity of Ti-Nb-based alloys. Mater. Sci. Eng. A 441, 112-118.

doi:10.1016/j.msea.2006.09.051

Hao, Y.L., Li, S.J., Sun, S.Y., Zheng, C.Y., Hu, Q.M., Yang, R., 2005. Super-elastic titanium alloy with unstable plastic deformation. Appl. Phys. Lett. 87, 091906.

doi:10.1063/1.2037192

Hao, Y.L., Li, S.J., Sun, S.Y., Zheng, C.Y., Yang, R., 2007. Elastic deformation behaviour of $\mathrm{Ti}-24 \mathrm{Nb}-4 \mathrm{Zr}-7.9 \mathrm{Sn}$ for biomedical applications. Acta Biomater. 3, 277-286. doi:10.1016/j.actbio.2006.11.002

Hao, Y.L., Niinomi, M., Kuroda, D., Fukunaga, K., Zhou, Y.L., Yang, R., Suzuki, A., 2002. Young's Modulus and Mechanical Properties of Ti-29Nb-13Ta-4.6Zr in Relation to $\alpha$ " Martensite. Metall. Mater. Trans. A 33, 3137-3144.

Hao, Y.L., Wang, H.L., Li, T., Cairney, J.M., Ceguerra, A.V., Wang, Y.D., Wang, Y.D., Wang, D., Obbard, E.G., Li, S.J., Yang, R., 2016. Superelasticity and Tunable Thermal Expansion across a Wide Temperature Range. J. Mater. Sci. Technol. 32, 705-709.

doi:10.1016/j.jmst.2016.06.017

Ho, W.F., Ju, C.P., Lin, J.H., 1999. Structure and properties of cast binary Ti-Mo alloys. Biomaterials 20, 2115-22.

Huang, C., Browne, D., McFadden, S., 2006. A phase-field simulation of austenite to ferrite transformation kinetics in low carbon steels. Acta Mater. 54, 11-21. doi:10.1016/j.actamat.2005.08.033

James, R.D., Zhang, Z., James, R.D., Zhang, Z., 2005. A Way to Search for Multiferroic Materials with "Unlikely" Combinations of Physical Properties, in: Planes, A., Manosa, L., Saxena, A. (Eds.), Magnetism and Structure in Functional Materials. Springer, Berlin, pp. 159-175. doi:10.1007/3-540-31631-0_9

Ji, Y., Ding, X., Lookman, T., Otsuka, K., Ren, X., 2013. Heterogeneities and strain glass behavior: Role of nanoscale precipitates in low-temperature-aged Ti48.7Ni51.3 alloys. Phys. Rev. B 87, 104110. doi:10.1103/PhysRevB.87.104110

Jin, Y.M., Artemev, A., Khachaturyan, A.G., 2001. Three-dimensional phase field model of lowsymmetry martensitic transformation in polycrystal: simulation of $\zeta^{\prime} 2$ martensite in $\mathrm{AuCd}$ alloys. Acta Mater. 49, 2309-2320. doi:10.1016/S1359-6454(01)00108-2

Khachaturyan, A.G., 1983. Theory of Structural Transformations in Solids. John Wiley \& Sons, New York.

Landau, L.D., Lifshitz, E.M., 1980. Statistical physics. Pergamon Press, Oxford.

Levitas, V., 2013. Phase-field theory for martensitic phase transformations at large strains. Int. J. Plast. 
Liu, J., Jin, M., Ni, C., Shen, Y., Fan, G., Wang, Z., Zhang, Y., Li, C., Liu, Z., Jin, X., 2011. Strain glassy behavior and premartensitic transition in Au7Cu5Al4 alloy. Phys. Rev. B 84, 140102. doi:10.1103/PhysRevB.84.140102

Liu, J., Wang, Y.Y., Hao, Y.-L., Wang, Y.Y., Nie, Z., Wang, D., Ren, Y., Lu, Z., Wang, J., Wang, H., Hui, X., Lu, N., Kim, M.J., Yang, R., 2013. New intrinsic mechanism on gumlike superelasticity of multifunctional alloys. Sci. Rep. 3, 2156. doi:10.1038/srep02156

Lloveras, P., Castán, T., Porta, M., Planes, A., Saxena, A., 2010. Thermodynamics of stressinduced ferroelastic transitions: Influence of anisotropy and disorder. Phys. Rev. B 81, 214105. doi:10.1103/PhysRevB.81.214105

Long, M., Rack, H.., 1998. Titanium alloys in total joint replacement - a materials science perspective. Biomaterials 19, 1621-1639. doi:10.1016/S0142-9612(97)00146-4

Lookman, T., Shenoy, S.R., Rasmussen, K.Ø., Saxena, A., Bishop, A.R., 2003. Ferroelastic dynamics and strain compatibility. Phys. Rev. B 67, 024114.

doi:10.1103/PhysRevB.67.024114

Lovey, F.C.C., Condo', A.M., Torra, V., Condó, a. M., Torra, V., 2004. A model for the interaction of martensitic transformation with dislocations in shape memory alloys. Int. J. Plast. 20, 309-321. doi:10.1016/S0749-6419(03)00081-0

Ma, N., Chen, Q., Wang, Y., 2006. Implementation of high interfacial energy anisotropy in phase field simulations. Scr. Mater. 54, 1919-1924. doi:10.1016/j.scriptamat.2006.02.005

Ma, N., Kazaryan, A., Dregia, S.A., Wang, Y., 2004. Computer simulation of texture evolution during grain growth: Effect of boundary properties and initial microstructure. Acta Mater. 52, 3869-3879. doi:10.1016/j.actamat.2004.05.001

Mackenzie, J.K., Bowles, J.S., 1954. The crystallography of martensite transformations II. Acta Metall. 2, 138-147. doi:10.1016/0001-6160(54)90103-0

Mamivand, M., Asle Zaeem, M., El Kadiri, H., 2014. Shape memory effect and pseudoelasticity behavior in tetragonal zirconia polycrystals: A phase field study. Int. J. Plast. 60, 71-86. doi:10.1016/j.ijplas.2014.03.018

Matsumoto, H., Watanabe, S., Hanada, S., 2005. Beta TiNbSn alloys with low young's modulus and high strength. Mater. Trans. 46, 1070-1078. doi:10.2320/matertrans.46.1070

MILITZER, M., MECOZZI, M., SIETSMA, J., VANDERZWAAG, S., 2006. Threedimensional phase field modelling of the austenite-to-ferrite transformation. Acta Mater. 54, 3961-3972. doi:10.1016/j.actamat.2006.04.029

Moelans, N., Blanpain, B., Wollants, P., 2008. An introduction to phase-field modeling of microstructure evolution. Calphad 32, 268-294. doi:10.1016/j.calphad.2007.11.003

Morris Jr., J.W., Hanlumyuang, Y., Sherburne, M., Withey, E., Chrzan, D.C., Kuramoto, S., Hayashi, Y., Hara, M., 2010. Anomalous transformation-induced deformation in $\langle 110\rangle$ textured Gum Metal. Acta Mater. 58, 3271-3280. doi:10.1016/j.actamat.2010.02.001

Norfleet, D.M., Sarosi, P.M., Manchiraju, S., Wagner, M.F.-X., Uchic, M.D., Anderson, P.M., Mills, M.J., 2009. Transformation-induced plasticity during pseudoelastic deformation in 
Ni-Ti microcrystals. Acta Mater. 57, 3549-3561. doi:10.1016/j.actamat.2009.04.009

Obbard, E.G., Hao, Y.L., Talling, R.J., Li, S.J., Zhang, Y.W., Dye, D., Yang, R., 2011. The effect of oxygen on $\alpha^{\prime \prime}$ martensite and superelasticity in Ti-24Nb-4Zr-8Sn. Acta Mater. 59, 112-125. doi:10.1016/j.actamat.2010.09.015

Otsuka, K., Ren, X., 2005. Physical metallurgy of Ti-Ni-based shape memory alloys. Prog. Mater. Sci. 50, 511-678. doi:10.1016/j.pmatsci.2004.10.001

Paranjape, H.M., Manchiraju, S., Anderson, P.M., 2016. A phase field - Finite element approach to model the interaction between phase transformations and plasticity in shape memory alloys. Int. J. Plast. 80, 1-18. doi:10.1016/j.ijplas.2015.12.007

Pelton, A.R., Huang, G.H., Moine, P., Sinclair, R., 2012. Effects of thermal cycling on microstructure and properties in Nitinol. Mater. Sci. Eng. A 532, 130-138. doi:10.1016/j.msea.2011.10.073

Pi, Z.P., Fang, Q.H., Liu, B., Feng, H., Liu, Y., Liu, Y.W., Wen, P.H., 2016. A phase field study focuses on the transverse propagation of deformation twinning for hexagonal-closed packed crystals. Int. J. Plast. 76, 130-146. doi:10.1016/j.ijplas.2015.08.002

Plancher, E., Tasan, C.C., Sandloebes, S., Raabe, D., 2013. On dislocation involvement in Ti-Nb gum metal plasticity. Scr. Mater. 68, 805-808. doi:10.1016/j.scriptamat.2013.01.034

Saito, T., Furuta, T., Hwang, J.-H., Kuramoto, S., Nishino, K., Suzuki, N., Chen, R., Yamada, A., Ito, K., Seno, Y., Nonaka, T., Ikehata, H., Nagasako, N., Iwamoto, C., Ikuhara, Y., Sakuma, T., 2003. Multifunctional Alloys Obtained via a Dislocation-Free Plastic Deformation Mechanism. Science 300, 464-7. doi:10.1126/science.1081957

Sarkar, S., Ren, X., Otsuka, K., 2005. Evidence for Strain Glass in the Ferroelastic-Martensitic System Ti50-xNi50+x. Phys. Rev. Lett. 95, 205702. doi:10.1103/PhysRevLett.95.205702

Song, Y., Chen, X., Dabade, V., Shield, T.W., James, R.D., 2013. Enhanced reversibility and unusual microstructure of a phase-transforming material. Nature 502, 85-8. doi:10.1038/nature12532

Wang, D., Hou, S., Wang, Y., Ding, X., Ren, S., Ren, X., Wang, Y., 2014. Superelasticity of slim hysteresis over a wide temperature range by nanodomains of martensite. Acta Mater. 66, 349-359. doi:10.1016/j.actamat.2013.11.022

Wang, D., Lv, D., Gao, Y., Wang, Y., Ren, X., Wang, Y., 2016. Defect strength and strain glass state in ferroelastic systems. J. Alloys Compd. 661, 100-109. doi:10.1016/j.jallcom.2015.11.095

Wang, D., Zhang, Z., Zhang, J., Zhou, Y., Wang, Y., Ding, X., Wang, Y., Ren, X., 2010. Strain glass in Fe-doped Ti-Ni. Acta Mater. 58, 6206-6215. doi:10.1016/j.actamat.2010.07.040

Wang, J., Sehitoglu, H., 2014. Martensite modulus dilemma in monoclinic NiTi-theory and experiments. Int. J. Plast. 61, 17-31. doi:10.1016/j.ijplas.2014.05.005

Wang, Y., Chen, L., Khachaturyan, A., 1992. Particle translational motion and reverse coarsening phenomena in multiparticle systems induced by a long-range elastic interaction. Phys. Rev. B. Condens. Matter. 
Wang, Y., Chen, L.-Q., Khachaturyan, A.., 1991. Shape evolution of a precipitate during straininduced coarsening. Scr. Metall. Mater. 25, 1387-1392. doi:10.1016/0956-716X(91)904192

Wang, Y., Gao, J., Wu, H., Yang, S., Ding, X., Wang, D., Ren, X., Wang, Y., Song, X., Gao, J., 2014. Strain glass transition in a multifunctional $\beta$-type Ti alloy. Sci. Rep. 4, 3995. doi:10.1038/srep03995

Wang, Y., Khachaturyan, A.G., 2006. Multi-scale phase field approach to martensitic transformations. Mater. Sci. Eng. A 438-440, 55-63. doi:10.1016/j.msea.2006.04.123

Wang, Y., Khachaturyan, A.G., 1997. Three-dimensional field model and computer modeling of martensitic transformations. Acta Mater. 45, 759-773. doi:10.1016/S1359-6454(96)00180-2

Wang, Y., Li, J., 2010. Phase field modeling of defects and deformation. Acta Mater. 58, 12121235. doi:10.1016/j.actamat.2009.10.041

Wang, Y.U., Jin, Y.M., Khachaturyan, A.G., 2002. Phase field microelasticity theory and modeling of elastically and structurally inhomogeneous solid. J. Appl. Phys. 92, 1351-1360. doi:10.1063/1.1492859

Wayman, C.M., 1994. The phenomenological theory of martensite crystallography: Interrelationships. Metall. Mater. Trans. A 25, 1787-1795. doi:10.1007/BF02649029

Wechsler, M.S., Lieberman, D.S., READ, T., 1953. On the theory of the formation of martensite. Trans. Am. Inst. Min. Metall. Eng. 197, 1503-1515.

Wen, Y.H., Wang, Y., Chen, L.Q., 2002. Coarsening dynamics of self-accommodating coherent patterns. Acta Mater. 50, 13-21. doi:10.1016/S1359-6454(01)00335-4

Yao, T., Du, K., Hao, Y., Li, S., Yang, R., Ye, H., 2016. In-situ observation of deformation induced $\alpha^{\prime \prime}$ phase transformation in a $\beta$-titanium alloy. Mater. Lett. 182, 281-284. doi:10.1016/j.matlet.2016.07.021

Yeddu, H.K., Lookman, T., Saxena, A., 2013. Strain-induced martensitic transformation in stainless steels: A three-dimensional phase-field study. Acta Mater. 61, 6972-6982. doi:10.1016/j.actamat.2013.08.011

Zarnetta, R., Takahashi, R., Young, M.L., Savan, A., Furuya, Y., Thienhaus, S., Maaß, B., Rahim, M., Frenzel, J., Brunken, H., Chu, Y.S., Srivastava, V., James, R.D., Takeuchi, I., Eggeler, G., Ludwig, A., 2010. Identification of Quaternary Shape Memory Alloys with Near-Zero Thermal Hysteresis and Unprecedented Functional Stability. Adv. Funct. Mater. 20, 1917-1923. doi:10.1002/adfm.200902336

Zhang, L., Wang, D., Ren, X., Wang, Y., 2015. A new mechanism for low and temperatureindependent elastic modulus. Sci. Rep. 5, 11477. doi:10.1038/srep11477

Zhang, S.Q., Li, S.J., Jia, M.T., Prima, F., Chen, L.J., Hao, Y.L., Yang, R., 2011. Low-cycle fatigue properties of a titanium alloy exhibiting nonlinear elastic deformation behavior. Acta Mater. 59, 4690-4699. doi:10.1016/j.actamat.2011.04.015

Zhang, Y.W., Li, S.J., Obbard, E.G., Wang, H., Wang, S.C., Hao, Y.L., Yang, R., 2011. Elastic properties of $\mathrm{Ti}-24 \mathrm{Nb}-4 \mathrm{Zr}-8 \mathrm{Sn}$ single crystals with bcc crystal structure. Acta Mater. 59, 
3081-3090. doi:10.1016/j.actamat.2011.01.048

Zhou, N., Lv, D.C., Zhang, H.L., McAllister, D., Zhang, F., Mills, M.J., Wang, Y., 2014.

Computer simulation of phase transformation and plastic deformation in IN718 superalloy: Microstructural evolution during precipitation. Acta Mater. 65, 270-286.

doi:10.1016/j.actamat.2013.10.069

Zhou, N., Shen, C., Mills, M.J., Wang, Y., 2008. Contributions from elastic inhomogeneity and from plasticity to $\gamma^{\prime}$ rafting in single-crystal Ni-Al. Acta Mater. 56, 6156-6173. doi:10.1016/j.actamat.2008.08.027

Zhou, Y., Xue, D., Ding, X., Wang, Y., Zhang, J., Zhang, Z., Wang, D., Otsuka, K., Sun, J., Ren, X., 2010. Strain glass in doped Ti50(Ni50-xDx) $(\mathrm{D}=\mathrm{Co}, \mathrm{Cr}, \mathrm{Mn})$ alloys: Implication for the generality of strain glass in defect-containing ferroelastic systems. Acta Mater. 58, 54335442. doi:10.1016/j.actamat.2010.06.019

Zysset, P.K., Edward Guo, X., Edward Hoffler, C., Moore, K.E., Goldstein, S. a., 1999. Elastic modulus and hardness of cortical and trabecular bone lamellae measured by nanoindentation in the human femur. J. Biomech. 32, 1005-1012. doi:10.1016/S00219290(99)00111-6

\section{Appendix A. Parameters of phase field model}

Coefficients of Landau polynomial in Eq. (9)

$A_{1}=9 \times(T-190) \times 10^{4} \mathrm{~J} / \mathrm{m}^{3}, A_{2}=13.93 \times 10^{7} \mathrm{~J} / \mathrm{m}^{3}, A_{3}=16.30 \times 10^{7} \mathrm{~J} / \mathrm{m}^{3}$

The Landau polynomial coefficients, $A_{1}=A_{0}\left(T-T_{0}\right), A_{2}$ and $A_{3}$, in the chemical free energy are chosen in such a way that they produce free energy curves at different temperatures shown in Fig. A.1, which yieds an athermal MT at the vicinity of $190 \mathrm{~K}$, which is consistent with the experimentally determined martensitic start temperature, Ms (Hao et al., 2006).

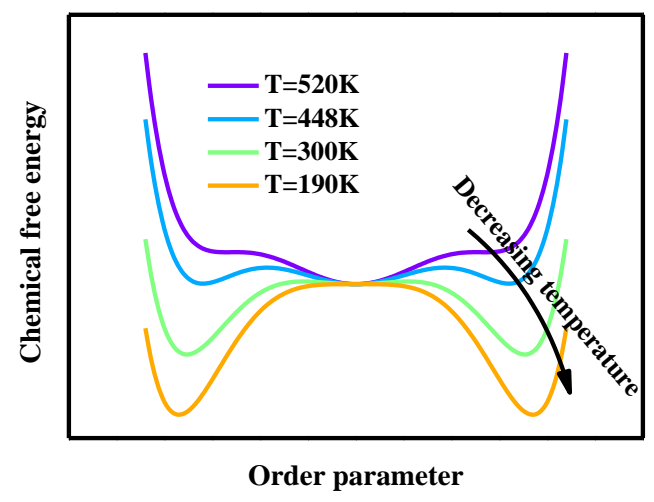


Fig. A.1 chemical free energy at different temperatures.

Gradient coefficient in Eq. (10)

$\beta=1.8 \times 10^{-12} \mathrm{~J} / \mathrm{m}$

Kinetic coefficient in Eq. (14)

$M=1 \times 10^{-4} \mathrm{~m}^{4} \mathrm{~J}^{-1} \mathrm{~s}^{-1}$ (Huang et al., 2006; Wang et al., 2014)

Amplitude of Langevin noise in Eq. (15)

$2 \frac{k_{B} T}{|\Delta f| l^{3}}=0.06$

\section{Appendix B. Category of twin planes}

Table B.1 Compound twin planes

\begin{tabular}{ccc}
\hline Variant pairs & $\boldsymbol{a}$ & $\boldsymbol{n}$ \\
\hline V1\&V2 & $(0,-0.0005,0.0430)$ & $(0,1,0)$ \\
& $(0,-0.0430,0.0005)$ & $(0,0,-1)$ \\
V3\&V4 & $(0.0430,0,-0.0005)$ & $(0,0,1)$ \\
& $(0.0005,0,-0.0430)$ & $(-1,0,0)$ \\
V5\&V6 & $(-0.0005,0.0430,0)$ & $(1,0,0)$ \\
& $(-0.0430,0.0005,0)$ & $(0,-1,0)$ \\
\hline
\end{tabular}

Table B.2 Type-I twin planes

\begin{tabular}{ccc}
\hline Variant pairs & $\boldsymbol{a}$ & $\boldsymbol{n}$ \\
\hline V1\&V3 & $(-0.0506,-0.0528,0.0301)$ & $(-1,1,0)$ \\
V1\&V4 & $(0.0506,-0.0528,0.0301)$ & $(1,1,0)$ \\
V1\&V5 & $(0.0506,-0.0301,0.0528)$ & $(1,0,-1)$ \\
\hline
\end{tabular}




\begin{tabular}{lll}
\hline V1\&V6 & $(-0.0506,-0.0301,0.0528)$ & $(-1,0,-1)$ \\
V2\&V3 & $(-0.0506,0.0528,0.0301)$ & $(-1,-1,0)$ \\
V2\&V4 & $(0.0506,0.0528,0.0301)$ & $(1,-1,0)$ \\
V2\&V5 & $(-0.0506,0.0301,0.0528)$ & $(-1,0,-1)$ \\
V2\&V6 & $(0.0506,0.0301,0.0528)$ & $(1,0,-1)$ \\
V3\&V5 & $(-0.0301,0.0506,0.0528)$ & $(0,1,-1)$ \\
V3\&V6 & $(-0.0301,-0.0506,0.0528)$ & $(0,-1,-1)$ \\
V4\&V5 & $(0.0301,-0.0506,0.0528)$ & $(0,-1,-1)$ \\
V4\&V6 & $(0.0301,0.0506,0.0528)$ & $(0,1,-1)$ \\
\hline
\end{tabular}

Table B.3Type-II twin planes

\begin{tabular}{ccc}
\hline Variant pairs & \multicolumn{1}{c}{$a$} & $n$ \\
\hline V1\&V3 & $(0.0553,-0.0574,0.0006)$ & $(0.6508,0.6508,-0.3911)$ \\
V1\&V4 & $(-0.0553,-0.0574,0.0006)$ & $(-0.6508,0.6508,-0.3911)$ \\
V1\&V5 & $(-0.0553,-0.0006,0.0574)$ & $(-0.6508,0.3911,-0.6508)$ \\
V1\&V6 & $(0.0553,-0.0006,0.0574)$ & $(0.6508,0.3911,-0.6508)$ \\
V2\&V3 & $(0.0553,0.0574,0.0006)$ & $(0.6508,-0.6508,-0.3911)$ \\
V2\&V4 & $(-0.0553,0.0574,0.0006)$ & $(-0.6508,-0.6508,-0.3911)$ \\
V2\&V5 & $(0.0553,0.0006,0.0574)$ & $(0.6508,-0.3911,-0.6508)$ \\
V2\&V6 & $(-0.0553,0.0006,0.0574)$ & $(-0.6508,-0.3911,-0.6508)$ \\
V3\&V5 & $(-0.0006,-0.0553,0.0574)$ & $(0.3911,-0.6508,-0.6508)$ \\
V3\&V6 & $(-0.0006,0.0553,0.0574)$ & $(0.3911,0.6508,-0.6508)$ \\
V4\&V5 & $(0.0006,0.0553,0.0574)$ & $(-0.3911,0.6508,-0.6508)$ \\
V4\&V6 & $(0.0006,-0.0553,0.0574)$ & $(-0.3911,-0.6508,-0.6508)$ \\
\hline
\end{tabular}

\section{Figure captions}


Fig. 1. Crystal lattice of (a) parent (BCC) and (b) martensitic (orthorhombic) phase, respectively. The structure outlined in blue in (a) notes the Bain correspondence between the parent and martensitic phases. Lattice parameters in this figure have a unit of angstrom.

Fig. 2. Schematic drawing of herringbone structures obtained from solutions of Eq. (5). $\mathrm{Vj}(j=1-6)$ is short for variant $j, \mathrm{C}$ denotes compound twin, and I and II represent type-I and type-II twins, respectively.

Fig. 3. Schematic drawing of a wedge-like self-accommodating microstructure consisting of 4 martensitic variants represented by the red, yellow, purple and blue colors. The austenite is represented by the green color.

Fig. 4. Phase field simulation of microstructure evolution during MT in Ti2448 at 193K. (a)-(h) are microstructures at reduced time of $10.8,15.6,18.6,21.6,24,33,105.6$, and 600, respectively.

Fig. 5. Volume fraction changes of six martensitic variants during MT in Ti2448 at 193K. The inset shows volume fraction changes at the beginning of the transformation.

Fig. 6. Polytwinned microstructures predicted by the phase field simulations at $193 \mathrm{~K}$. (a) Compound twin, (b) type-I twin, (c) type-II twins. (d) and (e) are two dimensional projections of (c).

Fig. 7. Phase field simulation prediction of two kinds of herringbone structures at $193 \mathrm{~K}$ : (a) C-II and (e) I-C. (b)-(d) and (f)-(h) are two dimensional projections of (a) and (e), respectively.

Fig. 8. HRSTEM image and diffraction pattern of a twin structure in Ti2448. Twin planes are marked with the dash lines, and the orientation of the TEM foil relative to the orientation of the crystal structure of the martensite is indicated by the insets.

Fig. 9 Two dimensional sections of three dimensional shear stress fields, $\sigma_{12}, \sigma_{13}, \sigma_{23}$, associated 
with (a) a single variant martensitic particle of around 60nm in (b)-(d) Ti2448 and (e)-(g) Ti$49.8 \mathrm{Ni}$.

Fig. 10 (a)-(c) are shear stress fields, $\sigma_{13}$, induced by (g) type I twin, (h) type II twin, and (i) C-II herringbone structure in Ti2448, respectively. (d)-(f) are shear stress fields corresponding to (j) type I twin, (k) type II twin, and (1) C-II herringbone structure in Ti-49.8Ni, respectively.

Fig. 11 Schematic drawings of (a) C-II and (b) I-C herringbone structures consisting of variants with non-equal volume fractions. $r$ denotes the volume fraction of a variant in a herringbone structure, which can be calculated via the edge length of variants as shown in (a) and (b).

Fig. 12 (a) the variation of strain state (indicated by the red arrows) of the C-II herringbone structure consisting of V1, V2, V3 and V4 in principle strain space and (b) the rotation of the principle strain axes of the I-C herringbone structure consists of V1, V2, V3 and V4 with respect to volume fraction $r$. The two red arrows in (a) indicate the strain states of the C-II herringbone structure corresponding to $r=0$ and $r=1$, respectively. The cyan, blue and purple sets of principle strain axes correspond to $\mathrm{r}=0,0.5$, and 1 , respectively. The blue solid dot in (a) correspond to the case of $r=0.5$. 